\title{
AN EXPLICIT FORMULA FOR THE ARITHMETIC GEOMETRIC MEAN IN GENUS 3
}

\author{
D. LEHAVI AND C. RITZENTHALER
}

\begin{abstract}
The arithmetic geometric mean algorithm for calculation of elliptic integrals of the first type was introduced by Gauss. The analog algorithm for Abelian integrals of genus 2 was introduced by Richelot (1837) and Humbert (1901). We present the analogous algorithm for Abelian integrals of genus 3 .
\end{abstract}

\section{INTRODUCTION}

1.1. The Arithmetic Geometric Mean (AGM) was discovered by Lagrange in 1785 and independently by Gauss in 1791. It is described as follows: given two positive numbers $a$ and $b$, define $M(a, b)$ as the limit of the following convergent sequences:

$$
\begin{array}{ll}
a_{0}:=a, & b_{0}=b \\
a_{n+1}=\frac{a_{n}+b_{n}}{2}, & b_{n+1}=\sqrt{a_{n} b_{n}} .
\end{array}
$$

During the period 1791-1799 Gauss discovered a relation between the AGM and elliptic curves:

1.2. Theorem (Gauss, see $\mathrm{Cox}, \mathrm{BM})$. For each pair of positive real numbers in the $A G M$ double sequence $a_{n}>b_{n}>0$ define:

$$
e_{n 1}:=\frac{1}{3}\left(a_{n}^{2}+b_{n}^{2}\right), \quad e_{n 2}:=e_{n 1}-b_{n}^{2}, \quad e_{n 3}:=e_{n 1}-a_{n}^{2} .
$$

Denote by $E_{n}$ the elliptic curve given by the equation

$$
y_{n}^{2}=4\left(x_{n}-e_{n 1}\right)\left(x_{n}-e_{n 2}\right)\left(x_{n}-e_{n 3}\right),
$$

then the following sequence of Abelian groups:

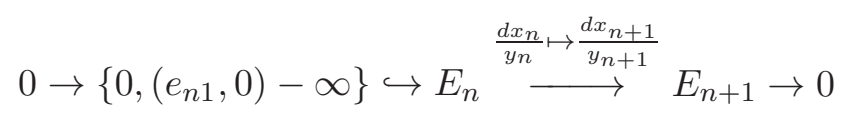

is exact for all $n$.

Date: September 6, 2018.

1991 Mathematics Subject Classification. 14H40,14H45,14Q05.

Key words and phrases. Prym varieties, Arithmetic Geometric Mean.

D. Lehavi was partially supported by Israel-US BSF grant 1998265.

C. Ritzenthaler acknowledges the financial support provided through the European Community's Human Potential Programme under contract HPRN-CT-2000-00114, GTEM. 
1.3. It is easy to see that we create in this way a sequence of 2 -isogenous elliptic curves. Gauss generalized the definition of the AGM to the complex numbers; in this case there is a choice involved when taking the square root. Gauss described the resulting correspondence, see Cox for the description. Recall that the real points in the Picard group of a curve which is defined over $\mathbb{R}$ are the divisor classes which are invariant under the action of the group $\operatorname{Gal}(\mathbb{C} / \mathbb{R})$ (see $G H$ sections $1-5)$. Gauss proved that if the 2-torsion points of $\operatorname{Pic}(E)$ are real then there is a unique $\alpha \in \operatorname{Pic}(E)[2]$ such that the 2-torsion points of $\operatorname{Pic}\left(E_{\alpha}^{\prime}\right)[2]$ are real. Applying this property iteratively, one gets an algorithm for calculating elliptic integrals (see $\mathrm{Cox},[\mathrm{BM}]$ ) of the form:

$$
\int_{e_{3}}^{e_{2}} \frac{d x}{\sqrt{\left(x-e_{1}\right)\left(x-e_{2}\right)\left(x-e_{3}\right)}} .
$$

This iterative algorithm is applied in numeric evaluations of certain types of Abelian integrals (see $[\mathrm{BB}]$ ).

In genera higher than 1 one can hope for an isogeny between the Jacobians of the curves. Before stating results in higher genera, we recall some facts on polarized Abelian varieties.

1.4. A pair $(A, \Theta)$ where $A$ is an Abelian variety and $\Theta$ is a divisor of $A$ is called a polarized Abelian variety. The divisor $\Theta$ is called the theta divisor of the polarized Abelian variety and the map $A \longrightarrow \operatorname{Pic}^{0}(A)$ defined by $a \mapsto T_{a}^{-1}(\Theta)-\Theta$ is called the polarization of the pair $(A, \Theta)$. Since the kernel of the polarization is a finite Abelian group whose rank is bounded by twice the genus of $A$ we describe its isomorphism type by 2 copies of a monotonic sequence of $\operatorname{genus}(A)$ natural numbers. We will abuse of notations and denote for instance the sequence $(2,2,2,2,1,1),(2,2,2,2,1,1)$ by $2^{4} 1^{2}$. If the polarization type is trivial we say that the polarization is principal; in this case we say that $A$ is a principally polarized Abelian or PPAV. The translates of $\Theta$ which contain 0 are called the theta characteristics of $A$. The theta characteristics are called even or odd if their multiplicity at 0 is even or odd. The theta characteristics of an Abelian variety $A$ induce a symplectic structure on the $\mathbb{F}_{2}$-vector space $A[2]$ (the group of 2-torsion points in $A$ ) in the following way: Let $\theta$ be a theta characteristic of $A$, then the map

$$
\begin{aligned}
q: A[2] & \longrightarrow \mathbb{F}_{2} \\
a & \mapsto h^{0}(\theta+a)+h^{0}(a) \bmod 2
\end{aligned}
$$

is a quadratic form over $\mathbb{F}_{2}$. The quadratic form $q$ induces the following symplectic pairing on the group $A[2]$ :

$$
\langle a, b\rangle=q(a+b)-q(a)-q(b) \bmod 2 .
$$

This pairing is called the Weil pairing. If $G$ is a subgroup of $A[2]$ we will use the notation

$$
G^{\perp}:=\{a \in A[2] \mid\langle a, g\rangle=0 \text { for all } g \in G\} .
$$


If $A$ is a PPAV and $G$ is a subgroup of the group $A[2]$, then $\operatorname{Pic}^{0}(A / G)$ is naturally isomorphic to $\operatorname{Pic}^{0}(A) / G^{\perp}$. Whence the Abelian variety $A / G$ is principally polarized only if $G$ is $0, A[2]$, or a maximal isotropic group of $A[2]$ with respect to the Weil pairing. Finally recall that a Jacobian of a smooth curve $C$ is principally polarized by the theta divisor $\Theta_{C}$ - the image of $\operatorname{Sym}^{g-1} C$ in $\operatorname{Jac}(C)$ under the Abel map.

1.5. The dimension of the moduli space of principally polarized Abelian varieties of genus $g$ is $g(g+1) / 2$, while the dimension of the moduli space of curves of genus $g \geq 2$ is $3 g-3$. Thus if $C$ is a generic curve of genus $g \leq 3$ and $L$ is a maximal isotropic subgroup of $\operatorname{Jac}(C)[2]$ then $\operatorname{Jac}(C) / L$ is a Jacobian of some curve $C^{\prime}$. This motivates the following questions:

- Is this an algebraic correspondence?

- Does there exist a curve $C^{\prime}$ for every pair $C, L$ in genera 2,3 (and not only generically)?

- If $C$ is a real curve, are there "distinguished" maximal subgroups ?

- What is the situation for genera higher then 3 ?

In the case $g=2$ the first three questions were settled by Richelot (see Ric]) and Humbert (see Hum). Both Richelot \& Humbert described an iterative algorithm for curves of genus 2 with 6 real Weierstrass points, Richelot described algebraically the curve $C^{\prime}$ in terms of the curve $C$ and Humbert described the isomorphism

$$
H^{0}\left(C, K_{C}\right) \longrightarrow H^{0}\left(C^{\prime}, K_{C^{\prime}}\right) .
$$

See DL section 4 for a modern review of the construction and BM for the resulting integration identities and the iterative integration algorithm resulting in the real case. Using modern tools (namely GAGA), the answer to the first question is immediately positive in all genera. Donagi \& Livné solved the second question for genus $g=3$, and answered negatively the last question:

1.6. Theorem (Donagi \& Livné, see DL). Let $C$ be a smooth curve of genus $g$ over a base field of characteristic different from 2,3. Let $L \subset \operatorname{Jac}(C)[2]$ be a maximal isotropic subgroup (with respect to the Weil pairing) then:

- If $g=3$ then there exists a curve $C^{\prime}$ such that $\operatorname{Jac}\left(C^{\prime}\right) \cong \operatorname{Jac}(C) / L$. The curve $C^{\prime}$ can be described algebraically in terms of the curve $C$ and the maximal isotropic subgroup $L$.

- If $g>3$ then generically there is no curve $C^{\prime}$ such that $\operatorname{Jac}\left(C^{\prime}\right) \cong$ $\operatorname{Jac}(C) / L$.

1.7. The proof that Donagi and Livné presented for genus 3 is constructive in the set-theoretic sense. However, as a basis for explicit work it has drawbacks; it is not clear how to give coordinates to the spaces and functions involved or how one can track the canonical classes.

The object of this paper is to extend Gauss's original work on curves of genus 1 to the case of genus 3. In Section 2 we use the Coble-Recillas 
construction (reviewed in 2.9 2.10) to give an alternative construction to the one proposed in DL; we describe the curve $C^{\prime}$ in terms of the pair $(C, L)$, where the curve $C$ is a generic curve. In Section 3 we describe the isomorphism between the canonical classes $\left|K_{C}\right|$ and $\left|K_{C^{\prime}}\right|$. In Section 4 we derive the formulas describing the curve $C^{\prime}$ in terms of the curve $C$ and the isomorphism $H^{0}\left(C, K_{C}\right) \cong H^{0}\left(C^{\prime}, K_{C^{\prime}}\right)$. We also show how to iterate the construction. In Section [5 we concentrate on real curves: assuming the curve $C$ is a real M-curve (i.e. a real curve with 4 components) we present a distinguished maximal isotropic subgroup of $\operatorname{Jac}(C)[2]$, resulting in an iterative integration algorithm.

Although our construction is stated over the complex numbers it is mostly algebraic. The complex structure is used in one crucial point: we use a result (due to Jordan in Jor section 332, or see Harris's modern approach in Har) stating that the Galois group of the bitangents of a smooth plane quartic over the complex numbers is $S P_{6}(2)$. In the rest of the paper we require only that the characteristic of the base field is the one arising from using the bigonal and trigonal constructions; the characteristic of the base field $K$ is not 2 or 3 (see the discussion in the introduction to [DL).

Some of the proofs in Sections 45 are computer aided proofs. The Mathematica and MAGMA programs which generated the computer part of the proofs appears in [LR].

1.8. We remark that the AGM has a nice application in the area of curves over finite fields: Mestre observed that the theta-function identities involved in the AGM over $p$-adic fields can be used to study the number of points in curves over finite fields. See Mes, LL for results elliptic and hyperelliptic results and Rit for results on non hyperelliptic genus 3 curves.

Acknowledgments. Section 2 and 3 of this paper were part of the first author's Ph.D. thesis, done under the advising of R. Livné, who suggested the series of questions above. I. Dolgachev kindly gave the first author an early copy of his preprint Dol2, and introduced him to the works of A. Coble.

\section{The COnstruction}

2.1. The idea behind our construction is to filter the level-2 data and perform the construction in three steps, using non principally polarized Abelian varieties to keep track of the level data. Throughout this paper we fix a generic curve $C$ of genus 3 , and a maximal isotropic flag $\mathcal{L}=\left(\langle\alpha\rangle=L_{1} \subsetneq\right.$ $\left.L_{2} \subsetneq L_{3}\right)$ with respect to the Weil pairing on $\operatorname{Jac}(C)[2]$. Let $C^{\prime}$ be a curve such that $\operatorname{Jac}\left(C^{\prime}\right) \cong \operatorname{Jac}(C) / L_{3}$. The flag $\mathcal{L}$ induces a dual isotropic flag $\mathcal{L}^{\prime}=\left(L_{1}^{\prime} \subsetneq L_{2}^{\prime} \subsetneq L_{3}^{\prime}\right)$ in $\operatorname{Jac}\left(C^{\prime}\right)[2]$ in the following way: $L_{1}^{\prime}$ (resp. $L_{2}^{\prime}$, resp. $\left.L_{3}^{\prime}\right)$ is the image of $L_{2}^{\perp}$ (resp. $L_{1}^{\perp}$, resp. $\operatorname{Jac}(C)[2]$ ) under the map $\operatorname{Jac}(C) \rightarrow \operatorname{Jac}(C) / L_{3}$. We denote by $\alpha$ (respectively $\alpha^{\prime}$ ) the non trivial element in $L_{1}$ (respectively $L_{1}^{\prime}$ ). Using the Coble-Recillas construction we will 
introduce ramified double covers $Y \longrightarrow E$ and $Y^{\prime} \longrightarrow E^{\prime}$ such that there are natural isomorphisms

$$
\operatorname{Prym}(Y / E) \cong \operatorname{Jac}(C) / L_{1}^{\perp}, \quad \operatorname{Prym}\left(Y^{\prime} / E^{\prime}\right) \cong \operatorname{Jac}\left(C^{\prime}\right) / L_{1}^{\prime \perp} .
$$

Using a bigonal construction (see Don p. 68-69 for an overview of the bigonal construction) we will prove that the polarized Abelian varieties $\operatorname{Prym}\left(Y^{\prime} / E^{\prime}\right)$ and $\operatorname{Prym}(Y / E)$ are dual to one another, up to finite data arising from $L_{2}$.

2.2. Before describing the geometry of the construction (in 2.10 2.17), we describe the finite symplectic algebra involved - the level-2 structure of the curve $C$. Define the following $S P_{6}(2)$ equivariant surjective map:

$$
\begin{gathered}
D:\left\{\begin{array}{c}
\text { unordered pairs of distinct } \\
\text { odd theta characteristics of } C
\end{array}\right\} \longrightarrow \operatorname{Jac}(C)[2] \backslash\{0\} \\
\left\{\theta_{1}, \theta_{2}\right\} \mapsto \theta_{1}-\theta_{2},
\end{gathered}
$$

(recall that the group $S P_{6}(2)$ acts on the odd theta characteristics via the monodromy action, see $\mathrm{Har}$ ). Since the group $S P_{6}(2)$ acts transitively on the set $\operatorname{Jac}(C)[2] \backslash\{0\}$, all the fibers of the map $D$ are of the same order: $\left(\begin{array}{c}28 \\ 2\end{array}\right) / 63=6$. For any 2-torsion point $\gamma$ in $\operatorname{Jac}(C)$ we define the Steiner system

$$
\Sigma_{\gamma}:=\cup_{x \in D^{-1}(\gamma)}\{x\} .
$$

Since the pairs in a fiber of the map $D$ do not intersect, the order of all the Steiner systems is 12 . In Propositions 2.3 and 2.4 below we discuss the relation between the symplectic structure on the vector space $\operatorname{Jac}(C)[2]$ and the combinatorics of the 63 Steiner systems.

2.3. Proposition. Let $\gamma, \gamma^{\prime}$ be two distinct elements in $\operatorname{Jac}(C)[2] \backslash\{0\}$, then $\#\left(\Sigma_{\gamma} \cap \Sigma_{\gamma^{\prime}}\right)$ is 4 if $\left\langle\gamma, \gamma^{\prime}\right\rangle=0$, and 6 otherwise.

Proof. Since the group $S P_{6}(2)$ acts transitively on pairs of distinct elements in $\operatorname{Jac}(C)[2] \backslash\{0\}$ with the same Weil pairing, the order of the set $\Sigma_{\gamma} \cap$ $\Sigma_{\gamma^{\prime}}$ depends only on the Weil pairing $\left\langle\gamma, \gamma^{\prime}\right\rangle$. We denote the two possible intersection orders by $n_{0}, n_{1}$. Any odd theta characteristic sits on $28-1=27$ different $\Sigma_{\gamma}$ s and the number of $\gamma \in \operatorname{Jac}(C)[2] \backslash\{0, \alpha\}$ such that $\langle\gamma, \alpha\rangle=0$ (resp. 1) is 30 (resp. 32). So we get

$$
\begin{aligned}
12 \cdot 27 & =\sum_{\alpha \neq 0} \#\left\{\theta \mid \theta \in \Sigma_{\alpha} \cap \Sigma_{\gamma}\right\} \\
& =\# \Sigma_{\alpha}+\#\{\alpha \mid\langle\gamma, \alpha\rangle=0\} n_{0}+\#\{\alpha \mid\langle\gamma, \alpha\rangle=0\} n_{1} \\
& =12+30 n_{0}+32 n_{1},
\end{aligned}
$$

and the unique non-negative integer solution of this equation is $n_{1}=6, n_{0}=$ 4 .

2.4. Proposition-Definition. The following properties hold: 
- Let $\gamma$ be a 2-torsion point in $\operatorname{Jac}(C)$ then the map

$$
\begin{aligned}
D_{\gamma}:\left\{\begin{array}{c}
\text { unordered pairs of distinct } \\
\text { classes in } \Sigma_{\gamma} / \gamma
\end{array}\right. & \longrightarrow \operatorname{Jac}(C)[2] / \gamma \\
\left\{a_{i}, a_{j}\right\} & \mapsto a_{i}-a_{j} .
\end{aligned}
$$

is an isomorphism on $\left(\gamma^{\perp} / \gamma\right) \backslash\{0\}$. Moreover, the map $D_{\gamma}$ maps the intersection pairing to the Weil pairing.

- Let $H$ be an isotropic subgroup of $\operatorname{Jac}(C)[2]$ of order 4 , then there is an unique theta characteristic $\theta$ such that for all $\alpha \in H, \theta+\alpha$ is an odd theta characteristic. Denoting the set $\{\theta+\alpha, \alpha \in H\}$ by $\Gamma_{H}$, one has $\Gamma_{H}=\cap_{\gamma \in H \backslash\{0\}} \Sigma_{\gamma}$.

- A maximal isotropic subgroup of $\operatorname{Jac}(C)[2]$ containing $\alpha$ is represented by a partition of $\Sigma_{\alpha} / \alpha$ in three pairs.

- If 7 Steiner systems intersect at 7 mutually distinct 4-tuples, then there is a maximal isotropic subgroup $G \subset \operatorname{Jac}(C)[2]$ such that these 7 Steiner systems are the Steiner systems of the non-zero elements of $G$.

Proof. Let $G_{\gamma} \subset S P_{6}(2)$ be the stabilizer of $\gamma$, then the $G_{\gamma}$ orbits of $\operatorname{Jac}(C)[2] / \gamma$ are the sets

$$
\{0\},\left(\gamma^{\perp} / \gamma\right) \backslash\{0\},\left(\operatorname{Jac}(C)[2] \backslash \gamma^{\perp}\right) / \gamma,
$$

which are of orders $1,15,16$ respectively. Since the map $D_{\gamma}$ is a non-trivial $G_{\gamma}$ equivariant map, and since the order of the set of unordered pairs of distinct points in $\Sigma_{\gamma}$ is $\left(\begin{array}{l}6 \\ 2\end{array}\right)=15$, the map $D_{\gamma}$ is a 1-1 map on the projective space $\left(\gamma^{\perp} / \gamma\right) \backslash\{0\}$. By a similar counting argument we prove the claim on the Weil pairing. The last three assertions follow from Proposition 2.3

2.5. Using the description of one maximal isotropic flag in terms of odd theta characteristics we describe the combinatorics of two isotropic flags: $\mathcal{L}^{\prime}=\left(\left\langle\alpha^{\prime}\right\rangle=L_{1}^{\prime} \subsetneq L_{2}^{\prime} \subsetneq L_{3}^{\prime}\right)\left(\right.$ see [2.1) and $\tilde{\mathcal{L}}=\left(\langle\tilde{\alpha}\rangle=\tilde{L}_{1} \subset \tilde{L}_{2} \subset \tilde{L}_{3}\right)$ in $\operatorname{Jac}\left(C^{\prime}\right)[2]$ such that:

$$
\tilde{L}_{1} \oplus L_{3}^{\prime}=L_{2}^{\prime \perp}, \quad \tilde{L}_{2} \oplus L_{3}^{\prime}=L_{1}^{\prime \perp}, \quad \tilde{L}_{3} \oplus L_{3}^{\prime}=\operatorname{Jac}\left(C^{\prime}\right)[2] .
$$

Such a description is essential for iterating the algorithm, as the pair $\left(C^{\prime}, \tilde{\mathcal{L}}\right)$ should be the starting point of the second iteration, playing the same role that the pair $(C, \mathcal{L})$ played in the first iteration. Note that $\tilde{\mathcal{L}}$ is not uniquely defined. For further uses, we will need the following lemma:

2.6. Lemma. For any subgroup $H \subset \tilde{L}_{3}$ of order 4 one has $\# \Gamma_{H} \cap \Sigma_{\alpha^{\prime}}=0$ if $H=\tilde{L}_{2}$ and $\# \Gamma_{H} \cap \Sigma_{\alpha^{\prime}}=2$ otherwise.

Proof. Since we have $\tilde{L}_{2} \subset L_{1}^{\prime \perp}$ the Weil pairings of the non trivial elements in $\tilde{L}_{2} / \tilde{L}_{1},\left(L_{1}^{\prime} \oplus \tilde{L}_{1}\right) / \tilde{L}_{1}$ are all 0. By Proposition 2.4 the intersection

$$
D_{\tilde{\alpha}}^{-1}\left(\tilde{L}_{2} / \tilde{L}_{1}\right) \cap D_{\tilde{\alpha}}^{-1}\left(\left(L_{1}^{\prime} \oplus \tilde{L}_{1}\right) / \tilde{L}_{1}\right)
$$

is empty. Whence, The intersection

$$
\Gamma_{\tilde{L}_{2}} \cap \Sigma_{\alpha^{\prime}}=\Gamma_{\tilde{L}_{2}} \cap\left(\Sigma_{\alpha^{\prime}} \cap \Sigma_{\tilde{\alpha}}\right)=\Gamma_{\tilde{L}_{2}} \cap \Gamma_{L_{1}^{\prime} \oplus \tilde{L}_{1}}
$$


is also empty. Reasoning in the same way, for any subgroup $H \subset \tilde{L}_{3}$ of order 4 such that $H \neq \tilde{L_{2}}$ we have $H$ is not a subset of $L_{1}^{\prime \perp}$. By Proposition 2.4

$$
\#\left(D_{\tilde{\alpha}}^{-1}\left(H / \tilde{L}_{1}\right) \cap D_{\tilde{\alpha}}^{-1}\left(\left(L_{1}^{\prime} \oplus \tilde{L}_{1}\right) / \tilde{L}_{1}\right)\right)=1
$$

and therefore the cardinality of the intersection $\Gamma_{H} \cap \Sigma_{\alpha^{\prime}}$ is 2 .

2.7. We now move to the geometric part of the construction. Recall that $C$ is a generic genus 3 curve and that $\mathcal{L}=\left(\langle\alpha\rangle=L_{1} \subsetneq L_{2} \subsetneq L_{3}\right)$ is a full isotropic flag in $\operatorname{Jac}(C)[2]$. We start with the Coble-Recillas construction. Next we construct the double cover $Y \longrightarrow E$ mentioned above and review its properties. Finally we describe the AGM construction through the bigonal construction.

2.8. Notations. If $V \rightarrow U$ is a cover without specific name for the morphism, we denote the morphism by $\pi_{V / U}$. In the following diagram we summarize some constructions and notations which will be introduced afterwords. Note that this diagram admit a symmetry with respect to the vertical axis passing through the right term. These symmetric objects, which are related to $\left(C^{\prime}, \mathcal{L}^{\prime}\right)$ under those constructions, will be denoted with '.

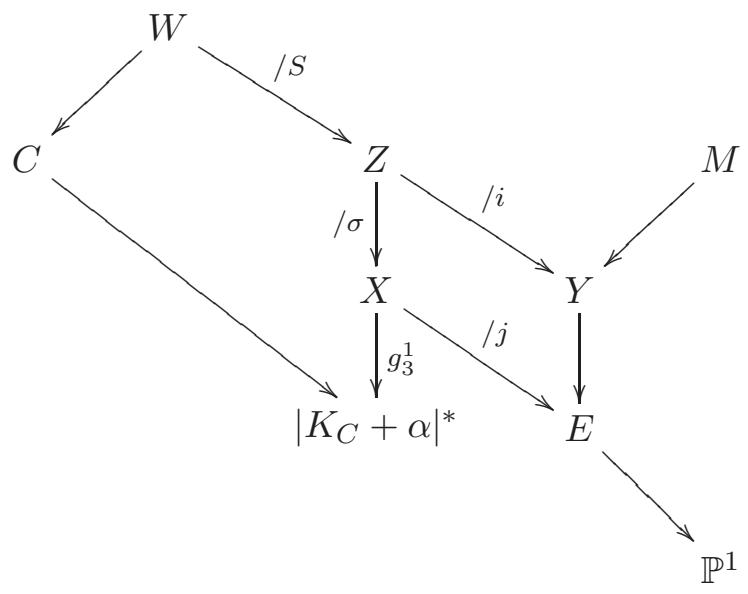

The left construction is Coble-Recillas trigonal construction, and the right construction is (half of) a bigonal construction (see Don p. 68-69).

2.9. Let us recall some elements of the theory of the trigonal construction (see for instance [Rec, [Dol1, [Leh]). Define

$$
W:=\overline{C \times\left|K_{C}+\alpha\right|^{*} C \backslash \Delta_{C}}=\left\{\left(p_{1}, p_{2}\right) \in C \times C \mid p_{1}+p_{2}<K_{C}+\alpha\right\}
$$

(where by $\Delta_{C}$ we denote the diagonal). The curve $W$ admits a natural involution - the coordinates switching which we denote by $S$. The curve $Z=W / S$ can be viewed as the subset of $\operatorname{Sym}^{2}(C)$ defined by $\left\{p_{1}+p_{2} \mid p_{1}+\right.$ $\left.p_{2}<K_{C}+\alpha\right\}$. The curve $Z$ admits three natural non-trivial involutions :

- $\sigma: p_{1}+p_{2} \mapsto p_{3}+p_{4}$ such that $p_{1}+p_{2}+p_{3}+p_{4} \sim K_{C}+\alpha$; denote $X:=Z / \sigma$.

- $i: p_{1}+p_{2} \mapsto p_{3}+p_{4}$ such that $p_{3}+p_{4} \sim p_{1}+p_{2}+\alpha$; denote $Y:=Z / i$. 
- $j=\sigma \circ i$; denote $F:=Z / j$.

Remark. The curves $Z$ has a "theta divisor interpretation": the Abel map Sym $^{2} C \longrightarrow \operatorname{Pic}^{2} C$ induces an isomorphism $Z \cong \Theta_{C} \cap\left(\Theta_{C}+\alpha\right)$ (see Leh 3.1-3.4). In particular, $Z$ is generically a smooth curve of genus 7 . Note that the involution $i$ is then $d \mapsto d+\alpha, j$ is $d \mapsto K_{C}-d$ and $\sigma$ is $d \mapsto K_{C}+\alpha-d$.

Let us denote by $\psi$ the morphism from $\operatorname{Sym}^{2}(C)$ to $\left|K_{C}\right|$ defined by sending $p_{1}+p_{2}$ to the line $\overline{p_{1} p_{2}}$. As the supports of $p_{1}+p_{2}$ and $j\left(p_{1}+p_{2}\right)$ as points on $C \subset\left|K_{C}\right|^{*}$ lie on the same line, $\psi$ induces a morphism from $F=Z / j$ to $\left|K_{C}\right|$ making the following diagram commutative:

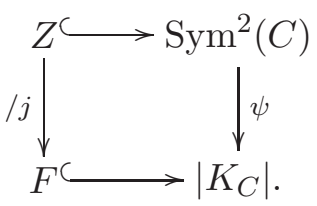

It is classical that bitangents to $C$ are in $1-1$ correspondence with odd theta characteristics. Moreover if $l$ is a bitangent corresponding to an element $\theta \in \Sigma_{\alpha}$, it defines a point on $Z$ (still denoted $\theta$ ): indeed, if $l \cdot C=2\left(p_{1}+p_{2}\right)$ and if $\theta+\alpha$ corresponds to a bitangent with divisor $2\left(p_{3}+p_{4}\right)$ one gets $\left(p_{1}+p_{2}\right)-\left(p_{3}+p_{4}\right) \sim \alpha$, so

$$
p_{1}+p_{2}+p_{3}+p_{4} \sim 2\left(p_{1}+p_{2}\right)+\alpha \sim K_{C}+\alpha .
$$

Thus $p_{1}+p_{2}<K_{C}+\alpha$.

By definition of $Z$ and $j$, the morphism $j$ is ramified exactly at the 12 elements of the Steiner system $\Sigma_{\alpha}$. As $Z$ is of genus $7, F$ is of genus 1 and by the preceding embedding the 12 bitangents (viewed as points in $\left|K_{C}\right|$ ) are points on $F$. Note that $F \subset\left|K_{C}\right|$ is a cubic: the degree of the map $F \longrightarrow\left|K_{C}\right|$ is 3 since $\operatorname{deg}(\psi)=6$, and the map is non degenerate because the points of $\psi\left(\Sigma_{\alpha}\right)$ are not colinear.

As $i$ is fixed point free and commutes with $j$, it defines a fixed point free involution $i_{F}$ on $F$ exchanging the points $\theta, \theta+\alpha \in \Sigma_{\alpha}$. This involution defines a point $\alpha_{F} \in \operatorname{Pic}^{0}(F)[2]$ such that for all $p \in F, p+\alpha_{F} \sim i_{F}(p)$. The quotient of $F$ by the involution $i_{F}$ is the curve $E=X / j$ (because $(Z / \sigma) / j=(Z / j) / i)$. Hence $E$ is naturally embedded in $\left|K_{C}\right|^{*}$ by the image of $\pi_{F / E}: p \mapsto p \cap\left(p+\alpha_{F}\right)$.

2.10. Theorem (Coble, see [Cob] sections 47-49, [Leh]). The images of the points of $\Sigma_{\alpha} \subset F$ under the map $\pi_{F / E}$ sit on a unique conic $Q \subset\left|K_{C}\right|^{*}$. They are the intersection points of the bitangents $\theta, \theta+\alpha \in \Sigma_{\alpha}$. The locus $Q \cap E$ is the ramification locus of the map $\pi_{Y / E}: Y \rightarrow E$.

2.11 (The folloing results are mostly due to Coble and to Recillas). Let $\alpha_{E}$ be the unique non zero element in $\left(\pi_{F / E}\right)_{*}(\operatorname{Pic}(F)[2]) \subset \operatorname{Pic}(E)[2]$. Given the double cover $Y \longrightarrow E$ and the 2-torsion point $\alpha_{E}$ one can reconstruct the curve $C$ and the linear system $\left|K_{C}+\alpha\right|$ in the following way. We have $F \simeq E / \alpha_{E}$. The curve $Z$ is isomorphic to the fibered product $Y \times_{E} F$. 
This construction induces two commuting involutions $i, j$ on $Z$. Since the double cover $\pi_{F / E}$ is unramified, the involutions $i, j$ on $Z$ are fixed points free. The genera of the curves $Z, X, Y$ are then $7,4,4$ respectively. The curve $X$ is a bielliptic curve of genus 4 which has only one $g_{3}^{1}$ up to the bielliptic involution. Thus we are back in the trigonal construction setting. Note that $C$ and the linear system $\left|K_{C}+\alpha\right|$ are invariant under the choice of the $g_{3}^{1}$. On our way to $\left(C^{\prime}, \mathcal{L}^{\prime}\right)$, we have now expressed $(\operatorname{Jac}(C), \alpha)$ in terms of the double cover $Z / X$. The second step in the construction (see Theorem 2.15) is to interpret the symplectic data through the quotient $i$. Our main tool for analysis of non principally polarized Abelian varieties are Lemmas 2.12 and 2.13 below.

2.12. Lemma (see [DL, Lemma 1). Let $\tilde{V} \longrightarrow V$ be an admissible double cover, and let $\nu \tilde{V} \longrightarrow \nu V$ be its partial normalization at $r>1$ points $x_{1}, \ldots, x_{r} \in V$. Let $g$ be the arithmetic genus of the partial normalization $\nu V$, so the arithmetic genus of $V$ is $g+r$. Then $\operatorname{Prym}(\tilde{V} / V)$ has a principal polarization, $\operatorname{Prym}(\nu \tilde{V} / \nu V)$ has a polarization of type $2^{g} 1^{r-1}$, and the pullback map

$$
\nu^{*}: \operatorname{Prym}(\tilde{V} / V) \longrightarrow \operatorname{Prym}(\nu \tilde{V} / \nu V)
$$

is an isogeny of degree $2^{r-1}$.

2.13. Lemma (the monodromy argument). Let $V^{\prime} \longrightarrow V$ be a finite cover such that the Galois group of the Galois closure of $V^{\prime} / V$ is 2-transitive on the cover, then the only section of the cover $V^{\prime} \times_{V} V^{\prime} \longrightarrow V$ is the diagonal.

2.14. We will apply lemma 2.13 with the covers $\mathcal{A}_{3}^{1} \rightarrow \mathcal{A}_{3}$ and $\mathcal{A}_{3}^{F} \rightarrow \mathcal{A}_{3}$ where $\mathcal{A}_{3}^{1}$ (resp. $\mathcal{A}_{3}^{F}$ ) is the moduli space of PPAV's dimension 3 with a 2 -torsion point (resp. with a maximal isotropic group).

2.15. Theorem. The quotient by $i$ induces an isogeny of Abelian varieties:

$$
\phi: \operatorname{Prym}(Z / X) \longrightarrow \operatorname{Prym}(Y / E) .
$$

Identifying the principally polarized Abelian varieties $\operatorname{Jac}(C)$ and $\operatorname{Prym}(Z / X)$ as in [Don] Theorem 2.11 (p. 76), the kernel of $\phi$ is $\alpha^{\perp}$.

Proof. The proof consists of three steps:

Step 1: The map $\phi: \operatorname{Prym}(Z / X) \longrightarrow \operatorname{Prym}(Y / E)$ is an isogeny :

To prove this claim it suffices to prove that the induced map on the tangent spaces at 0 is an isomorphism. We do this by considering the space $M:=H^{0}\left(Z, \Omega_{Z}^{1}\right)$ as a $\operatorname{Gal}(Z / E)$ module, and calculating the module decomposition to irreducible representations. We denote by $M_{-}$the irreducible representation corresponding to the character whose kernel is the subgroup $\langle-\rangle \subset \operatorname{Gal}(Z / E)$, and by $M_{1}$ the irreducible representation corresponding 
to the trivial character. Using these notations we have:

$$
\begin{aligned}
H^{0}\left(Z, \Omega_{Z}^{1}\right) & =M_{1} \oplus M_{i} \oplus M_{j} \oplus M_{\sigma}, \quad H^{0}\left(E, \Omega_{E}^{1}\right)=M_{1} \\
H^{0}\left(F, \Omega_{F}^{1}\right) & =M_{1}^{j} \oplus M_{i}^{j} \oplus M_{j}^{j} \oplus M_{\sigma}^{j}=M_{1} \oplus M_{j}, \\
H^{0}\left(Y, \Omega_{Y}^{1}\right) & =M_{1}^{i} \oplus M_{i}^{i} \oplus M_{j}^{i} \oplus M_{\sigma}^{i}=M_{1} \oplus M_{i}, \\
H^{0}\left(X, \Omega_{X}^{1}\right) & =M_{1}^{\sigma} \oplus M_{i}^{\sigma} \oplus M_{j}^{\sigma} \oplus M_{\sigma}^{\sigma}=M_{1} \oplus M_{\sigma} .
\end{aligned}
$$

However, since $E$ and $F$ are both of genus 1 , the map $H^{0}\left(F, \Omega_{F}^{1}\right) \longrightarrow$ $H^{0}\left(E, \Omega_{E}^{1}\right)$ is an isomorphism. Thus we have $M_{j}=0$, and our claim holds. Step 2: The kernel of the isogeny $\phi$ is a subset of $\operatorname{Prym}(Z / X)[2]$ :

Denoting by $[2]_{-}$the multiplication by 2 on an Abelian variety we have

$$
\pi_{Z / Y_{*}} \pi_{Z / Y}^{*}: \operatorname{Jac}(Y) \longrightarrow \operatorname{Jac}(Y)=[2]_{\operatorname{Jac}(Y)} .
$$

Denote by $\mu_{*}, \mu^{*}$ the restrictions of maps $\pi_{Z / Y_{*}}, \pi_{Z / Y}^{*}$ to the Abelian varieties $\operatorname{Prym}(Y / E), \operatorname{Prym}(Z / X)$ respectively. Note that $\phi=\mu^{*}$. We have $\mu^{*} \mu_{*}=[2]_{\operatorname{Prym}(Z / X)}$.

Step 3: Computation of the kernel of $\phi$ :

By applying Lemma 2.12 to some degeneration of the cover $Y / E$ along its ramification locus $Q \cap E$, on finds that the polarization type of the variety $\operatorname{Prym}(Y / E)$ is $2^{1} 1^{5}$. Thus, the order of the kernel of $\phi$ is 32 .

By Don Theorem 2.11 (p. 76) the norms in the trigonal construction induce an isomorphism $\operatorname{Jac}(C) \cong \operatorname{Prym}(Z / X)$. The kernel of $\phi$ is can thus be identified with $\beta^{\perp}$ for some $\beta \in \operatorname{Jac}(C)[2]$. The map $(\operatorname{Jac}(C), \alpha) \rightarrow$ $(\operatorname{Jac}(C), \beta)$ gives a endomorphism of $\mathcal{A}_{3}^{1}$ and then a section from $\mathcal{A}_{3}^{1}$ to $\mathcal{A}_{3}^{1} \times \mathcal{A}_{3} \mathcal{A}_{3}^{1}$. By Lemma 2.13 this section maps into the diagonal component so $\alpha=\beta$.

2.16. Denote by $q_{1}, \ldots, q_{6}$ the intersection points of $E$ and $Q$. In Proposition 2.4 we identified pairs of $q_{i}$ s with the non-zero points of the symplectic space $\alpha^{\perp} / \alpha$. This identification induces bijections between the following sets of data:

\begin{tabular}{l|l|l}
$\begin{array}{l}\text { data on isotropic subgroups in } \\
\operatorname{Jac}(C)[2] \text { that contain } \alpha .\end{array}$ & $\begin{array}{l}\text { data on isotropic } \\
\text { subgroups of } \alpha^{\perp} / \alpha .\end{array}$ & $\begin{array}{l}\text { partitions of the } \\
\text { points }\left\{q_{i}\right\}_{i=1 \ldots 6}\end{array}$ \\
\hline \hline Iso. subgps of order 4 & Iso. subgps of order 2 & $2+4$ \\
\hline Maximal iso. subgps & Maximal iso. subgps. & $2+2+2$ \\
\hline Full isotropic flags & Full isotropic flags & $2+(2+2)$
\end{tabular}

We let $\left\{q_{1}, q_{2}\right\},\left\{\left\{q_{3}, q_{4}\right\},\left\{q_{5}, q_{6}\right\}\right\}$ be the partition of the $q_{i}$ s that corresponds to the full isotropic flag $\mathcal{L}$. Denote by $\pi_{E / \mathbb{P}^{1}}$ the linear system $\left|q_{1}+q_{2}\right|$ on the curve $E$ and by $B$ the ramification locus of $\pi_{E / \mathbb{P}^{1}}$. Note that the symmetric construction introduces a set $B^{\prime}$. Using these definitions we are ready to prove the correctness of our construction: 
2.17. Theorem. Let assume that the ramification pattern of the tower $Y \longrightarrow$ $E \longrightarrow \mathbb{P}^{1}$ is generic. Denote by $\tilde{H} \longrightarrow H \longrightarrow \mathbb{P}^{1}$ the image of the tower $Y \longrightarrow E \longrightarrow \mathbb{P}^{1}$ by the bigonal construction. Then the tower $Y^{\prime} \longrightarrow E^{\prime} \longrightarrow$ $\mathbb{P}^{1}$ is the normalization of the tower $\tilde{H} \longrightarrow H \longrightarrow \mathbb{P}^{1}$. Moreover, there is a 1-1 correspondence between points $b \in Q \cap E \backslash\left\{q_{1}, q_{2}\right\}$ and points $b^{\prime} \in B^{\prime}$, given by

$$
\pi_{E / \mathbb{P}^{1}}(b)=\pi_{E^{\prime} / \mathbb{P}^{1}}\left(b^{\prime}\right) .
$$

Proof. The ramification pattern of the bigonal construction on $Y \longrightarrow E \longrightarrow$ $\mathbb{P}^{1}$ is the following (see Don p. 68-69):

- If $\pi_{E / \mathbb{P}^{1}}^{-1}(a)=\left\{q_{1}, q_{2}\right\}$, then $Y / E$ is ramified over both $q_{1}, q_{2}, \pi_{H / \mathbb{P}^{1}}^{-1}(a)$ is a node, and over this node the curve $\tilde{H}$ is a gluing of two ramified sheets (symbolically : $\subset \subset /=\mid \supset / \times)$.

- If $\pi_{E / \mathbb{P}^{1}}^{-1}(a)=2 b$ for some $b \in B$ then $Y / E$ is étale over $b, \pi_{H / \mathbb{P}^{1}}$ is étale over $a$, and $\tilde{H}$ is ramified over one of the points in $\pi_{H / \mathbb{P}^{1}}^{-1}(a)$ and ramified over the other one (symbolically : $\subset \subset / \subset \mid \subset=/=$ ).

- If $\pi_{E / \mathbb{P}^{1}}^{-1}(a) \ni q$ for some $q \in Q \cap E \backslash\left\{q_{1}, q_{2}\right\}$ then $Y / E$ is ramified over $q$, and étale over the other point in $\pi_{E / \mathbb{P}^{1}}^{-1}(a)$. Moreover, $\pi_{H / \mathbb{P}^{1}}$ is ramified at $a$, and $\tilde{H} / H$ is étale over both branches (symbolically $: \subset=/=\mid \subset \subset / \subset)$.

- In all other points the ramification patterns of the towers $Y / E / \mathbb{P}^{1}$ and $\tilde{H} / H / \mathbb{P}^{1}$ are generic (i.e. unramified).

Denote by $\nu \tilde{H}, \nu H$ the normalizations of the curves $\tilde{H}, H$ respectively. By the Riemann-Horowitz formula, the genera of the curves $\nu \tilde{H}, \nu H$ are 4,1 respectively. The ramification pattern over the points $\pi_{E / \mathbb{P}^{1}}\left(q_{3}\right), \pi_{E / \mathbb{P}^{1}}\left(q_{4}\right)$, $\pi_{E / \mathbb{P}^{1}}\left(q_{5}\right), \pi_{E / \mathbb{P}^{1}}\left(q_{6}\right)$ in the tower $Y \longrightarrow E \longrightarrow \mathbb{P}^{1}$ is $\subset=/=$. Thus, the partition $\left\{\left\{q_{3}, q_{4}\right\},\left\{q_{5}, q_{6}\right\}\right\}$ induces a partition to two pairs of the 4 ramification points of the map $\nu H \longrightarrow \mathbb{P}^{1}$, which induces a choice of a 2-torsion point in $\operatorname{Pic}^{0}(\nu H)$. Applying the reconstruction technique from 2.11 to the double cover $\nu \tilde{H} \longrightarrow \nu H$ and the 2 -torsion point we get a smooth curve $C^{\prime \prime}$ of genus 3. We claim that we have the following degrees for the isogenies

$$
\operatorname{Jac}(C) \stackrel{2}{\rightarrow} \operatorname{Prym}(\tilde{H} / H) \stackrel{2}{\rightarrow} \operatorname{Prym}(\nu \tilde{H} / \nu H) \stackrel{2}{\rightarrow} \operatorname{Jac}\left(C^{\prime \prime}\right)
$$

By $\mathrm{Pan}$ Proposition 3.1 (page 307) the Abelian variety $\operatorname{Prym}(\tilde{H} / H)$ is isomorphic to the dual of the Abelian variety $\operatorname{Prym}(Y / E)$. Since $\operatorname{Prym}(Y / E)$ is isomorphic to $\operatorname{Jac}(C) / \alpha^{\perp}$, we have by 1.4 that $\operatorname{Prym}(\tilde{H} / H) \simeq \operatorname{Jac}(C) / \alpha$. The second arrow is a consequence of Lemma 2.12 for the normalization of $\tilde{H} / H$ over the point of type $\supset / \times$. The third arrow follow from Theorem 2.15

Thus we have obtained an isogeny of degree $2^{3}$. By our observation in 1.4 this isogeny is given by a maximal isotropic group $L \in \operatorname{Jac}(C)[2]$. In the same spirit as in the proof of 2.15 we can use the monodromy argument of 
Lemma 2.13 for $L_{3}$ and $L$ to prove that $L=L_{3}$. Thus $C^{\prime \prime} \simeq C^{\prime}$ and we get

$$
\nu \tilde{H} \simeq Y^{\prime}, \quad \nu H \simeq E^{\prime} .
$$

The ramification patterns prove the last assertion of the theorem.

Remark. In the same way, one can describe analogous results for the other non-generic ramification patterns of the tower $Y \longrightarrow E \longrightarrow \mathbb{P}^{1}$, but this effort is redundant: in Section 4 we will find a formula which gives $C^{\prime}$ in terms of a generic pair $(C, L)$; since $C^{\prime}$ is continuous in the pair $(C, L)$, the formula will be correct for all pairs $(C, L)$ for which the denominators in the formula are non zero.

\section{THE ISOMORPHISM BETWEEN THE CANONICAL CLASSES}

3.1. In this section we describe the isomorphism $k:\left|K_{C^{\prime}}\right|^{*} \longrightarrow\left|K_{C}\right|^{*}$ between the duals of the canonical classes of the curves $C$ and $C^{\prime}$. In Section 4. this description is used to calculate the equation of the canonical embedding of curve $C^{\prime}$ in terms of the canonical embedding of the curve $C$ and to describe $\bar{k}: H^{0}\left(K_{C}\right) \rightarrow H^{0}\left(K_{C^{\prime}}\right)$.

We describe the isomorphism $k$ by considering the images and preimages (under the map $k$ ) of the sets $B, B^{\prime}$ (recall the definition in 2.16) and the points defined below:

$$
p:=E \cap \overline{q_{1} q_{2}} \backslash\left\{q_{1}, q_{2}\right\}, \quad p^{\prime}:=E^{\prime} \cap \overline{q_{1}^{\prime} q_{2}^{\prime}} \backslash\left\{q_{1}^{\prime}, q_{2}^{\prime}\right\} .
$$

The resulting description is encoded in the following theorem:

3.2. Theorem. The isomorphism $k$ is completely determined by the following identities:

$$
\begin{array}{cl}
k\left(Q^{\prime} \cap E^{\prime} \backslash\left\{q_{1}^{\prime}, q_{2}^{\prime}\right\}\right)=B, & k\left(\overline{q_{1}^{\prime} q_{2}^{\prime}}\right)=\overline{q_{1} q_{2}} \\
k\left(B^{\prime}\right)=Q \cap E \backslash\left\{q_{1}, q_{2}\right\}, & k\left(p^{\prime}\right)=p,
\end{array}
$$

where the identification of $Q^{\prime} \cap E^{\prime} \backslash\left\{q_{1}^{\prime}, q_{2}^{\prime}\right\}$ with $B$, and of $B^{\prime}$ with $Q \cap E \backslash$ $\left\{q_{1}, q_{2}\right\}$ are the ones from Theorem 2.17 .

Proof. The theorem follows from Theorem 3.6 and two applications of Theorem 3.4 below.

3.3. To describe the isomorphism $k$ we present it as a composition of three isomorphisms. Denote by $j_{Y}$ (resp. $J_{Y}$ ) the involution on the curve $Y$ (resp. the homology group $H^{0}\left(K_{Y}\right)$ ) induced from the double cover $Y \longrightarrow E$. Denote by $H^{0}\left(K_{Y}\right)_{\text {odd }}$ (resp. $H^{0}\left(K_{Y}\right)_{\text {even }}$ ) the odd (resp. even) part of $H^{0}\left(K_{Y}\right)$ with respect to the involution $J_{Y}$. We denote by $\left|K_{Y}\right|_{\text {odd }}$ (respectively $\left.\left|K_{Y}\right|_{\text {even }}\right)$ the projectivization of the vector space $H^{0}\left(K_{Y}\right)_{\text {odd }}$ (respectively $\left.H^{0}\left(K_{Y}\right)_{\text {even }}\right)$. We use the analog notations for subspaces of $\left|K_{Y^{\prime}}\right|$. The involution $J_{Y}$ induces an involution on the dual of the canonical system $\left|K_{Y}\right|^{*}$. The fixed set under this involution is the union of the projective 
plane $\left|K_{Y}\right|_{\text {odd }}^{*}$ and a point $p_{Y}$, the projectivization of the space $H^{0}\left(K_{Y}\right)_{\text {even }}$. By Theorem 2.17 we have a sequence of isogenies of Abelian varieties

$$
\operatorname{Jac}(C) \stackrel{/ \alpha^{\perp}}{\longrightarrow} \operatorname{Prym}(Y / E) \longrightarrow \operatorname{Prym}\left(Y^{\prime} / E^{\prime}\right) \stackrel{/ \alpha^{\prime \perp}}{\longleftarrow} \operatorname{Jac}\left(C^{\prime}\right) .
$$

Taking the tangents spaces at 0 of these varieties we get a sequence of isomorphisms:

$$
H^{0}\left(K_{C}\right) \stackrel{\bar{\phi}}{\longrightarrow} H^{0}\left(K_{Y}\right)_{\text {odd }} \stackrel{\bar{\psi}}{\longrightarrow} H^{0}\left(K_{Y^{\prime}}\right)_{\text {odd }} \stackrel{\overline{\phi^{\prime}}}{\longleftarrow} H^{0}\left(K_{C^{\prime}}\right),
$$

Taking the dual, inverse and projectivizations of the spaces and morphisms in Equation (11) we get another sequence of isomorphisms:

$$
\left|K_{C}\right|^{*} \stackrel{\phi}{\longrightarrow}\left|K_{Y}\right|_{\text {odd }}^{*} \stackrel{\psi}{\longrightarrow}\left|K_{Y^{\prime}}\right|_{\text {odd }}^{*} \stackrel{\phi^{\prime}}{\longleftarrow}\left|K_{C^{\prime}}\right|^{*} .
$$

By construction, these two morphisms have interpretation in terms of the trigonal and bigonal constructions. With the notations of 2.9 the morphism $\phi$ is induced by $\pi_{W / Y_{*}} \pi_{W / C}^{*}$. In the same way, denoting by $M$ the normalization of the Galois closure of the tower $Y \longrightarrow E \longrightarrow \mathbb{P}^{1}$, the isomorphism

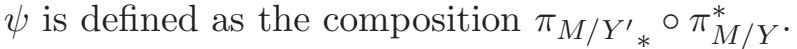

We discuss the morphism $\phi$ in Theorem 3.4 below. Our analysis is based on the two views of the set $\left\{q_{i}\right\}_{i=1 \ldots 6}$ presented in Theorem 2.10.

- The $q_{i}$ s are in natural 1-1 correspondence with intersection points of pairs of bitangents, which lie in $\left|K_{C}\right|^{*}$.

- The $q_{i}$ s are in natural 1-1 correspondence with the fixed points in $Y$ of the involution $j_{Y}$, i.e. with the points of $Y \cap\left|K_{Y}\right|_{\text {odd }}^{*} \subset\left|K_{Y}\right|^{*}$.

We interpret the relation between these views using the norms in the trigonal construction. If $\pi_{V / U}: V \longrightarrow U$ is one of the covers arising in our construction, we denote the ramification divisor of the map $\pi_{V / U}$ by $R_{V / U}$. We will make repetitive use of the following version of the Riemann-Horowitz theorem (see Har Proposition IV.2.1): Let $\omega$ be a differential on $U$ then the zero divisor of the differential $\pi_{V / U}^{*} \omega$ is $\pi_{V / U}^{*}\left((\omega)_{0}\right)+R_{V / U}$.

3.4. Theorem. The map $\phi:\left|K_{C}\right|^{*} \longrightarrow\left|K_{Y}\right|_{\text {odd }}^{*}$ takes each of the $q_{i}$ s to the corresponding point in $Y \cap\left|K_{Y}\right|_{\text {odd }}^{*} \subset\left|K_{Y}\right|^{*}$. Moreover, this property defines $\phi$.

Proof. To avoid confusion between the points of $W, Z, Y$ and divisors, we denote here a point of $W$ by $\left(p_{1}, p_{2}\right)$, on $Z$ by $\left(p_{1}+p_{2}\right)$ and a point of $Y$ by $\left\{\left(p_{1}+p_{2}\right),\left(p_{3}+p_{4}\right)\right\}$ if $p_{3}+p_{4}=j\left(p_{1}+p_{2}\right) \in Z$.

Let $\left\{p_{1}+p_{2}, p_{3}+p_{4}\right\}$ be the pair of theta characteristics in $\Sigma_{\alpha}$ which represent one of the $q_{i}$ s (see 2.16). Let $\omega \in H^{0}\left(K_{C}\right)$ be a differential such that $\omega_{0}=2\left(p_{1}+p_{2}\right)$ then

$$
\left(\pi_{W / C}^{*}(\omega)\right)_{0}-R_{W / C}=\pi_{W / C}^{*}\left(\omega_{0}\right)=2\left(\left(p_{1}, p_{2}\right)+\left(p_{2}, p_{1}\right)+\sum_{\substack{1 \leq i \leq 2 \\ 3 \leq j \leq 4}}\left(p_{i}, p_{j}\right)\right) .
$$


So $\left(\pi_{W / C}^{*}(\omega)\right) \geq 2\left(\left(p_{1}, p_{2}\right)+\left(p_{2}, p_{1}\right)\right)+R_{W / C}$. By the properties of the trigonal construction (see Don p. 74) we have $R_{W / C} \geq R_{W / Z}$. So we get

$$
\left(\pi_{W / C}^{*}(\omega)+S \pi_{W / C}^{*}(\omega)\right)_{0} \geq 2\left(\left(p_{1}, p_{2}\right)+\left(p_{2}, p_{1}\right)\right)+R_{W / Z}
$$

The left summand is precisely the pull back of $\pi_{W / Z_{*}} \pi_{W / C}^{*}(\omega)$ so finally get

$$
\left(\pi_{W / Z_{*}} \pi_{W / C}^{*}(\omega)\right)_{0} \geq 2\left(p_{1}+p_{2}\right) .
$$

Now

$$
\pi_{Z / Y_{*}} \pi_{W / Z_{*}} \pi_{W / C}^{*}(\omega)=\pi_{W / Y_{*}} \pi_{W / C}^{*}(\omega)=\bar{\phi}(\omega) \in H^{0}\left(K_{Y}\right)_{\text {odd }}
$$

So by invariance under $J_{Y}$

$$
(\bar{\phi}(\omega))_{0}=\left(\pi_{W / Y_{*}} \pi_{W / C}^{*}(\omega)\right)_{0} \geq 2\left\{\left(p_{1}+p_{2}\right),\left(p_{3}+p_{4}\right)\right\} .
$$

As $q_{i}$ is the intersection points of the two bitangents supported by $\left(p_{1}, p_{2}\right),\left(p_{3}, p_{4}\right)$, the last inequality implies

$$
\phi\left(q_{i}\right)=\left\{\left(p_{1}+p_{2}\right),\left(p_{3}+p_{4}\right)\right\} .
$$

Since the $q_{i}$ s are 6 non-collinear points this property completely describes the map $\phi$.

3.5. It remains to analyze the isogeny $\psi$ induced from the bigonal construction relating the double covers $Y \longrightarrow E$ and $Y^{\prime} \longrightarrow E^{\prime}$ (see the proof of Theorem 2.17). We make the identifications of Theorem 3.4 (so $\psi$ becomes $k$ ). As the linear system $\left|p+q_{1}+q_{2}\right|$ spans the space $\left|K_{Y}\right|_{\text {odd }}^{*}$ (and the same with the symmetric notation ') we reduce the description of the map $\mathbb{P} \psi$ to a description of a natural isomorphism between these linear systems on the curves $E, E^{\prime}$.

3.6. Theorem. The isomorphism $\psi$ is determined by the following identities:

$$
\begin{array}{ll}
\psi\left(Q^{\prime} \cap E^{\prime} \backslash\left\{q_{1}^{\prime}, q_{2}^{\prime}\right\}\right)=B, & \psi\left(\overline{q_{1}^{\prime} q_{2}^{\prime}}\right)=\overline{q_{1} q_{2}} \\
\psi\left(B^{\prime}\right)=Q \cap E \backslash\left\{q_{1}, q_{2}\right\}, & \psi\left(p^{\prime}\right)=p,
\end{array}
$$

where the identification of $Q^{\prime} \cap E^{\prime} \backslash\left\{q_{1}^{\prime}, q_{2}^{\prime}\right\}$ with $B$, and of $B^{\prime}$ with $Q \cap E \backslash$ $\left\{q_{1}, q_{2}\right\}$ are the ones from theorem 2.17.

Proof. The theorem follows from the two claims below:

(1) Let $t$ be a point in $\mathbb{P}^{1}$ and let $\omega$ be a differential in $H^{0}\left(K_{Y}\right)_{\text {odd }}$ such that $\frac{1}{2} \pi_{Y / E_{*}}\left((\omega)_{0}\right)=p+\pi_{E / \mathbb{P}^{1}}^{*}(t)$ then the image of $\omega$ under $\bar{\psi}$ satisfies $\frac{1}{2} \pi_{Y^{\prime} / E^{\prime}}{ }_{*}\left((\bar{\psi}(\omega))_{0}\right)=p^{\prime}+\pi_{E^{\prime} / \mathbb{P}^{1}}^{*}(t)$.

(2) Let $b$ be a point in the set $B \subset E$ and let $q_{i}^{\prime}$ be the corresponding point (in the sense of Theorem 2.17) in the set $Q^{\prime} \cap E^{\prime}$. Let $\omega$ be a differential in $H^{0}\left(K_{Y}\right)_{\text {odd }}$ such that $\pi_{Y^{\prime} / E^{\prime}}{ }_{*}(\omega)_{0} \geq 2 b$ then the image of $\omega$ under $\bar{\psi}$ satisfies $(\bar{\psi}(\omega))_{0} \geq 2 q_{i}^{\prime}$.

As in the proof of Theorem 3.4 we make repetitive use of Riemann-Horowitz theorem. 
Proof of claim 1: Let $\omega$ be a differential as in the first claim above. Since the zero divisor of the differential $\omega$ is moving with $t$, the intersection $\left(\pi_{M / Y}^{*}(\omega)\right)_{0} \cap R_{M / Y}$ is generically empty. By the definition of the bigonal construction

$$
\begin{aligned}
(\bar{\psi}(\omega))_{0} & =\left(\pi_{M / Y^{\prime}{ }_{*}} \pi_{M / Y}^{*}(\omega)\right)_{0}=\frac{1}{2} \pi_{M / Y^{\prime}{ }_{*}}\left(\left(\pi_{M / Y}^{*}(\omega)\right)_{0}-R_{M / Y}\right) \\
& =\frac{1}{2} \pi_{M / Y^{\prime}{ }_{*}} \pi_{M / Y}^{*}\left(\pi_{Y / \mathbb{P}^{1}}{ }^{*}(t)+\pi_{Y / E}{ }^{*}(p)\right) .
\end{aligned}
$$

Then

$\frac{1}{2} \pi_{Y^{\prime} / E^{\prime}{ }_{*}}\left((\bar{\psi}(\omega))_{0}\right)=\frac{1}{2}\left(\pi_{M / E^{\prime}{ }_{*}} \pi_{M / \mathbb{P}^{1}}^{*}(t)+\pi_{M / E^{\prime}{ }_{*}} \pi_{M / E}^{*}(p)\right)=\pi_{E / \mathbb{P}^{1}}^{*}(t)+p^{\prime}$.

Proof of claim 2: Define the objects $\omega, b, q_{i}^{\prime}$ as in the second claim above. By the definition of the point $b$ we have $\left(\pi_{M / Y}^{*}(\omega)\right)_{0} \geq \pi_{M / \mathbb{P}^{1}}^{*}\left(\pi_{E / \mathbb{P}^{1}}(b)\right)$. Whence:

$$
\begin{aligned}
(\bar{\psi}(\omega))_{0} & =\left(\pi_{M / Y^{\prime}{ }_{*}} \pi_{M / Y}^{*}(\omega)\right)_{0}=\frac{1}{2} \pi_{M / Y^{\prime}}\left(\left(\pi_{M / Y}^{*}(\omega)\right)_{0}-R_{M / Y^{\prime}}\right) \\
& \geq \frac{1}{2} \pi_{M / Y^{\prime}{ }_{*}}\left(\left\{t \in \pi_{M / \mathbb{P}^{1}}^{*}\left(\pi_{E / \mathbb{P}^{1}}(b)\right) \mid t \notin R_{M / Y^{\prime}}\right\}\right) .
\end{aligned}
$$

To prove the inequality $(\bar{\psi}(\omega))_{0} \geq 2 q_{i}$ it suffices to show that $R_{M / Y^{\prime}} \cap$ $\pi_{M / Y^{\prime}}^{*} R_{Y^{\prime} / E^{\prime}}=\emptyset$. By the bigonal construction dictionary (see Theorem [2.17), if the cover $Y \longrightarrow \mathbb{P}^{1}$ is ramified over a point $t$ then the cover $E^{\prime} \longrightarrow \mathbb{P}^{1}$ is étale over $t$. Since the curve $M$ can be defined as the product $E^{\prime} \times_{\mathbb{P}^{1}} Y$ there are no multiple points in the ramification divisor $R_{M / \mathbb{P}^{1}}$. This proves that $R_{M / Y^{\prime}} \cap \pi_{M / Y^{\prime}}^{*} R_{Y^{\prime} / E^{\prime}}=\emptyset$.

\section{A small matter of programming}

4.1. In the previous sections we presented an explicit construction of the AGM in genus 3. In this section we close the gap between "explicit" and a formula. We tackle five problems: describing the pair $(C, \alpha)$ (4.3.4.4), describing the intermediate data $(E, Q),\left(E^{\prime}, Q^{\prime}\right)$ in term of $\left(C, L_{2}\right)$ 4.5. 4.13), describing the pair $\left(C^{\prime}, \mathcal{L}^{\prime}\right)$ in terms of the pair $(C, \mathcal{L})$ (4.144.17), describing the isomorphism $\bar{k}: H^{0}\left(C, K_{C}\right) \longrightarrow H^{0}\left(C^{\prime}, K_{C^{\prime}}\right)$ (4.18), and describing a flag $\tilde{\mathcal{L}}$ (see 2.5) in terms of $E^{\prime}, Q^{\prime}, \mathcal{L}^{\prime}$ (4.19 4.21). We solve these problems by a "coordenification" of the proof of Theorem 3.2 We identify the two spaces $\left|K_{C}\right|^{*}$ and $\left|K_{C^{\prime}}\right|^{*}$ under the isomorphism $k$. We denote the coordinates on this space by $x, y, z$ (a precise choice of coordinate is described in 4.3).

4.2. Notations. To write the equations, we use the lexicographic order on the dual coordinates of $x, y, z$. i.e. instead of writing $a x^{2}+b x y+c x z+$ $d y^{2}+e y z+f z^{2}$, we will write $(a, b, c, d, e, f)$. If we will talk about the corresponding curve, we will use the projective coordinates $(a ; b ; c ; d ; e ; f)$. Finally, we will abuse the notations by using the name of a curve in $\mathbb{P}^{2}$ for its defining equation. 
4.3. The data $(C, \alpha)$. Let us consider the natural bilinear map

$$
\begin{aligned}
H^{0}\left(K_{C}+\alpha\right) \times H^{0}\left(K_{C}+\alpha\right) & \longrightarrow H^{0}\left(2 K_{C}\right)=H^{0}\left(\mathcal{O}_{\left|K_{C}\right|}(2)\right) \\
\left(\left(a s_{1}+b s_{2}\right),\left(c s_{1}+d s_{2}\right)\right) & \mapsto a c A_{1}+(b c+a d) A_{3}+b d A_{2}
\end{aligned}
$$

defined by the tensor multiplication of the sections $s_{i}$. Identifying $\left|K_{C}+\alpha\right|$ with $\mathbb{P}^{1}$ we also get a map

$$
m: \mathbb{P}^{1} \times \mathbb{P}^{1} \rightarrow\left|2 K_{C}\right| .
$$

We simplify the conics $A_{i}$ by a special choice of our sections $s_{i}$ : Let $\theta_{i}, \theta_{i}+\alpha \in$ $\Sigma_{\alpha}$ for $i=1,2$; we assume below that the pair $\left(\theta_{i}, \theta_{i}+\alpha\right)$ corresponds to the points $q_{i}$ by Theorem 2.10. Note that the choice of a distinguished pair $\left\{q_{1}, q_{2}\right\}$ is equivalent to the choice of $L_{2}$ in $\operatorname{Jac}(C)[2]$ as described in [2.9] Let $l_{1 i}$ (resp. $l_{2 i}$ ) the bitangents corresponding to $\theta_{i}$ (resp. $\left.\theta_{i}+\alpha\right)$ and let be $D_{j i}$ the effective divisor of degree 2 such that $2 D_{j i}=\left(l_{j i}\right)_{0}$, the we have

$$
K_{C}+\alpha \sim D_{1 i}+\sigma\left(D_{1 i}\right) \sim D_{1 i}+i \circ j\left(D_{1 i}\right) \sim D_{1 i}+i\left(D_{1 i}\right) \sim D_{1 i}+D_{2 i} .
$$

We denote by $s_{i}$ the sections of $H^{0}\left(K_{C}+\alpha\right)$ corresponding to $D_{1 i}+D_{2 i}$. With this choice of sections, we have $A_{1}=l_{11} l_{21}$ and $A_{2}=l_{12} l_{22}$. We now fix the coordinates $(x ; y ; z)$ of $\left|K_{C}\right|^{*}$ such that $A_{1}=(y-z)(y+z)$ and $A_{2}=(x-z)(x+z)$. The following Proposition is now a particular case of a classical result (see Dol2]).

4.4. Proposition. The quartic $C$ is given by $A_{3}^{2}-A_{1} A_{2}=0$. Assume that the quadrics $A_{1}, A_{2}, A_{3}$ are respectively given by

$$
(0,0,0,1,0,-1), \quad(1,0,0,0,0,-1), \quad(a, b, c, d, e, f),
$$

then the coordinates of $C \in\left|\mathcal{O}_{\left|K_{C}\right|^{*}}(4)\right|$ are

$\left(a^{2} ; 2 a b ; 2 a c ; b^{2}+2 a d-1 ; 2 b c+2 a e ; 1+c^{2}+2 a f ; 2 b d ; 2 c d+2 b e ; 2 c e+2 b f ;\right.$

$\left.; 2 c f ; d^{2} ; 2 d e ; 1+e^{2}+2 d f ; 2 e f ; f^{2}-1\right)$.

In 4.54.13, we will find the equations for the intermediate data $(E, Q),\left(E^{\prime}, Q^{\prime}\right)$.

4.5. Theorem. Assume that the quadrics $A_{1}, A_{2}, A_{3}$ are as in 4.4. The coordinates of the curves $E, E^{\prime} \in\left|\mathcal{O}_{\left|K_{C}\right|^{*}}(3)\right|$ are

$$
\begin{aligned}
E= & (0 ; c ; b ; e ; 2(a+d+f) ; e ; 0 ; b ; c ; 0), \\
E^{\prime}= & \left(-2 a c ;-2 a e ; b^{2}-c^{2}-4 a f-1-4 a^{2} ; 2 c d ; 4 b(d-a) ; 2 b e-4 a c-2 c f\right. \\
& \left.; 2 d e ; 1-b^{2}+4 d^{2}+e^{2}+4 d f ; 2 e(2 d+f)-2 b c ; e^{2}-c^{2}\right) .
\end{aligned}
$$


The coordinates of the conics $Q, Q^{\prime} \in\left|\mathcal{O}_{\left|K_{C}\right|^{*}}(2)\right|$ are

$$
\begin{aligned}
Q= & \left(0 ; c e\left(b^{2}-1+4 a d\right)-2 b\left(c^{2} d+e^{2} a\right)\right. \\
& ; b\left(-2 b c d-e+b^{2} e\right)+2 a\left(b^{2} c+c e^{2}-2 b e f\right)-4 a^{2} b e ; 0 \\
& ; b^{3} c+2 c^{2} d e+2 b^{2}(d-a) e-b c\left(1+4 d^{2}+4 d f\right) \\
& \left.; c^{2} e^{2}+b^{2}\left(c^{2}+e^{2}\right)-2 b c e(a+d+f)\right), \\
Q^{\prime}= & \left(-a\left(e^{2}-c^{2}\right) ; 0 ;(d-a)(c(a+d+f)-b e) ; d\left(c^{2}-e^{2}\right)\right. \\
& \left.; 2(a-d)(e(a+d+f)-b c) ;(d-a)\left(c^{2}-e^{2}\right)\right) .
\end{aligned}
$$

4.6. The essence of the proof is to convert the problem to a sequence of "steps" of the following form: find a pencil that is spanned by two known forms, and that contains another form we have to calculate. We perform these steps explicitly using a computer. Let us stress though, that in most cases two of the three forms involved in the computation are divisible by a known linear form. Thus, the obstinate reader could still check the computations below, up to and including Theorem 4.17. by hand.

The curve $E$. Recall (see 2.9] that to any point $p_{1}+p_{2} \in Z$ one associate a point $q \in E \subset\left|K_{C}\right|^{*}$ as $\overline{p_{1} p_{2}} \cap \overline{p_{3} p_{4}}$ where $i\left(p_{1}+p_{2}\right)=p_{3}+p_{4} \in Z$. Denote by $A$ the conic given by the product of the lines $\overline{p_{1} p_{2}}$ and $\overline{p_{3} p_{4}}$. Note that the singular point of $A$ is $q$.

4.7. Lemma. There exists a unique couple of sections (up to permutation) $\left(t_{1}, t_{2}\right) \in H^{0}\left(K_{C}+\alpha\right)^{2}$ such that $t_{1} t_{2}=A$. Conversely, the singular point of each singular conic in $m\left(\mathbb{P}^{1} \times \mathbb{P}^{1}\right)$ is on $E$.

Proof. Let us show the first assertion. By definition of $j$, the zero divisor of $A$ is $2 K_{C} \sim p_{1}+p_{2}+j\left(p_{1}+p_{2}\right)+p_{3}+p_{4}+j\left(p_{3}+p_{4}\right)$. As $p_{3}+p_{4}=i\left(p_{1}+p_{2}\right)$, one gets

$$
(A)_{0}=p_{1}+p_{2}+j \circ i\left(p_{1}+p_{2}\right)+i\left(p_{1}+p_{2}\right)+\sigma \circ i\left(p_{1}+p_{2}\right) .
$$

As $p_{1}+p_{2}+\sigma\left(p_{1}+p_{2}\right) \in\left|K_{C}+\alpha\right|\left(\right.$ resp. $\left.i\left(p_{1}+p_{2}\right)+\sigma \circ i\left(p_{1}+p_{2}\right) \in\left|K_{C}+\alpha\right|\right)$, the divisor defines a unique section $t_{1}$ (resp. $\left.t_{2}\right)$ in $H^{0}\left(K_{C}+\alpha\right)$.

To account for the permutation of the two lines in Lemma 4.7 we introduce the map

$$
\begin{aligned}
& v_{2}: \quad \mathbb{P}^{1} \times \mathbb{P}^{1} \quad \rightarrow \mathbb{P}^{2} \simeq \mathrm{Sym}^{2} \mathbb{P}^{1} \\
& (\lambda, \mu),\left(\lambda^{\prime}, \mu^{\prime}\right) \mapsto\left(\lambda \lambda^{\prime}, \mu \mu^{\prime}, \lambda \mu^{\prime}+\mu \lambda^{\prime}\right) .
\end{aligned}
$$

Let $(X ; Y ; Z)$ be the coordinates in $v_{2}\left(\mathbb{P}^{1} \times \mathbb{P}^{1}\right)$. Lemma 4.7 can be reformulated into : the curve $E$ can be seen as the locus $(x ; y ; z)$ of singular points in the net of conics $X A_{1}+Y A_{2}+Z A_{3}$. If $M=X_{0} A_{1}+Y_{0} A_{2}+Z_{0} A_{3}$ is such a conic, it is singular at $q_{0}$ if and only if

$$
\left(\begin{array}{l}
0 \\
0 \\
0
\end{array}\right)=\left(\begin{array}{c}
M_{x}\left(q_{0}\right) \\
M_{y}\left(q_{0}\right) \\
M_{z}\left(q_{0}\right)
\end{array}\right)=\left(\begin{array}{lll}
\left(A_{1}\right)_{x}\left(q_{0}\right) & \left(A_{1}\right)_{y}\left(q_{0}\right) & \left(A_{1}\right)_{z}\left(q_{0}\right) \\
\left(A_{2}\right)_{x}\left(q_{0}\right) & \left(A_{2}\right)_{y}\left(q_{0}\right) & \left(A_{2}\right)_{z}\left(q_{0}\right) \\
\left(A_{3}\right)_{x}\left(q_{0}\right) & \left(A_{3}\right)_{y}\left(q_{0}\right) & \left(A_{3}\right)_{z}\left(q_{0}\right)
\end{array}\right)^{t} \cdot\left(\begin{array}{c}
X_{0} \\
Y_{0} \\
Z_{0}
\end{array}\right) .
$$


Denote by $\operatorname{Jac}\left(A_{1}, A_{2}, A_{3}\right)$ the previous matrix. Thus the curve $E$ is given by $\operatorname{det}\left(\operatorname{Jac}\left(A_{1}, A_{2}, A_{3}\right)\right)=0$.

The curve $Q$. It is easy to check algebraically that the point $o:=(0 ; 0 ; 1)$ lies on $E$ (see 4.15 for a geometric explanation). Let $\hat{q}_{1}:=\overline{o q_{1}} \cap E \backslash\left\{o, q_{1}\right\}$ and $\hat{q_{2}}:=\overline{o q_{2}} \cap E \backslash\left\{o, q_{2}\right\}$. Let

$$
\hat{Q}:=\operatorname{Nulls}\left(4 \frac{\partial A_{3}}{\partial x} \frac{\partial A_{3}}{\partial y}-\frac{\partial A_{1}}{\partial y} \frac{\partial A_{2}}{\partial x}\right) .
$$

4.8. Proposition. The cubics E, $Q \overline{q_{1} \hat{q_{2}}}, \hat{Q} \overline{q_{1} q_{2}}$ lie on the same pencil. Moreover $p \in \overline{\hat{q_{1}} \hat{q_{2}}}$.

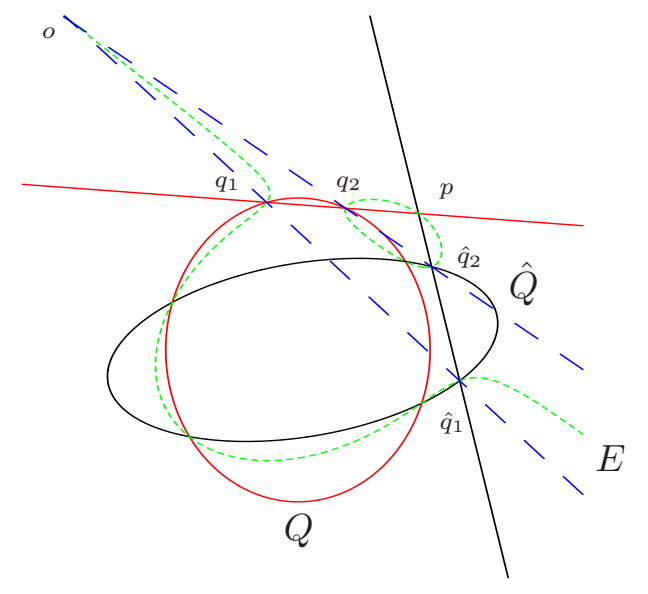

Proof. Recall that the intersection points $q_{i}$ of $Q \cap E$ are the intersection points of the pairs of bitangents $\theta_{i}, \theta_{i}+\alpha$. With the notations of the previous proof, if $p_{1}+p_{2}$ is the divisor associated to $\theta_{i}$, the divisor associated to the product of the two lines is $2\left(p_{1}+p_{2}\right)+2 i\left(p_{1}+p_{2}\right)$. So $t_{1}=t_{2}$ and the points $q_{i}$ correspond to singular points of singular conics of the form $m((\lambda ; \mu),(\lambda ; \mu))$ i.e.

$Q \cap E=\left\{(x ; y ; z) \mid \operatorname{Jac}\left(A_{1}, A_{2}, A_{3}\right)^{t} \cdot\left(\lambda^{2}, \mu^{2}, 2 \lambda \mu\right)^{t}=0\right.$ for one $\left.(\lambda ; \mu) \in \mathbb{P}^{1}\right\}$.

Since $\frac{\partial A_{2}}{\partial x}=\frac{\partial A_{1}}{\partial y}=0$ the points of $Q \cap E$ are the $(x ; y ; z)$ coordinates for which the following system admits a solution

$$
\begin{array}{lr}
\frac{\partial A_{2}}{\partial x} \mu^{2}+2 \frac{\partial A_{3}}{\partial x} \lambda \mu=0, & \frac{\partial A_{1}}{\partial y} \lambda^{2}+2 \frac{\partial A_{3}}{\partial y} \lambda \mu=0 \\
\frac{\partial A_{1}}{\partial z} \lambda^{2}+\frac{\partial A_{2}}{\partial z} \mu^{2}+2 \frac{\partial A_{3}}{\partial z} \lambda \mu=0, & (\lambda, \mu) \neq(0,0) .
\end{array}
$$

If $\lambda=0$ (resp. $\mu=0$ ) this system admits $q_{1}$ (resp. $q_{2}$ ) as a solution. If $\lambda \mu \neq 0$ then a solution satisfies

$$
\left(\frac{\partial A_{2}}{\partial x} ;-2 \frac{\partial A_{3}}{\partial x}\right)=(\lambda ; \mu)=\left(-2 \frac{\partial A_{3}}{\partial y} ; \frac{\partial A_{1}}{\partial y}\right),
$$


so it belongs to $\hat{Q}$. Hence the intersection points of $\hat{Q}$ and $Q$ are $E \cdot Q \backslash$ $\left\{q_{1}, q_{2}\right\}=B^{\prime}$ (see 3.2).

It follows from the definition of $\hat{q}_{i}$ that $\hat{q}_{i} \in \hat{Q}$ for $i=1,2$. We compute the intersections:

$$
E \cdot\left(\hat{Q \hat{q_{1}} \hat{q_{2}}}\right)>\left(B^{\prime}+q_{1}+q_{2}\right)+\hat{q_{1}}+\hat{q_{2}}
$$

and

$$
E \cdot\left(\hat{Q} \overline{q_{1} q_{2}}\right)>\left(B^{\prime}+\hat{q_{1}}+\hat{q_{2}}\right)+\left(q_{1}+q_{2}+p\right) .
$$

These 3 cubics have thus 8 points in common so they lie in the same pencil. Moreover their last intersection point is the same too, so $p \in \overline{q_{1}} \hat{q_{2}}$.

4.9. The curve $E^{\prime}$. For the purpose of describing the isotropic subgroup $L_{3}$, as well as for technical reasons we set the following notations: Denote by $Q_{p}$ the unique conic such that $Q_{p} \cdot E=2 p+B$, and by $Q_{p}^{\prime}$ the unique conic such that $Q_{p}^{\prime} \cdot E^{\prime}=2 p+B^{\prime}$ (the notations $B, B^{\prime}, p$ were defined in 2.16 and 3.1). Recall that under the identification of the linear system give by $k$, we have $E \cap Q \cap Q_{p}^{\prime}=B^{\prime}$ and $E^{\prime} \cap Q^{\prime} \cap Q_{p}=B$.

4.10. Proposition. The plane cubics $E, Q T_{p}(E), \overline{q_{1} q_{2}} Q_{p}^{\prime}$ lie on the same pencil.

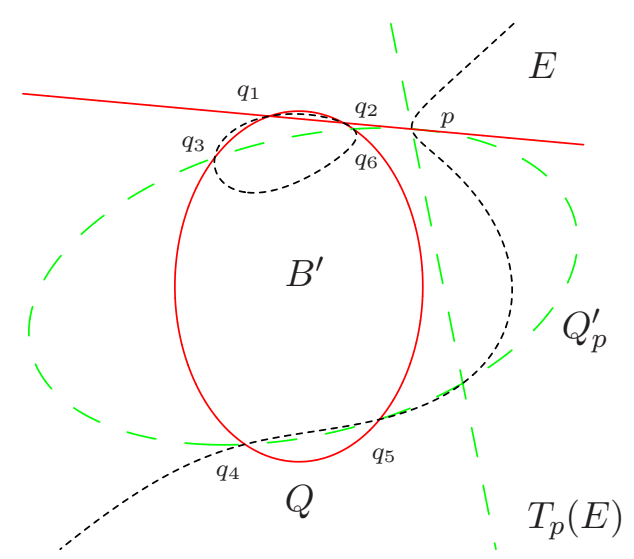

Proof. As in the previous proof, this follows after calculating the intersections:

$E \cdot\left(Q+T_{p}(E)\right)>\left(B^{\prime}+q_{1}+q_{2}\right)+2 p, \quad E \cdot\left(\overline{q_{1} q_{2}} Q_{p}^{\prime}\right)>\left(q_{1}+q_{2}+p\right)+\left(B^{\prime}+p\right)$.

4.11. Let $J=\cup_{b \in B^{\prime}} \overline{p b}$; we compute the quartic defining $J$ using the following procedure.

(1) Since $p=(-e ; c ; 0)$, the lines passing through $p$ are given by linear forms $c x+e y-\alpha z=0$ for some $\alpha \in \mathbb{C}$. Thus $J=\prod_{i=1}^{4}\left(c x+e y-\alpha_{i}\right)$, where the $\alpha_{i}$ 's are defined by the property $c x_{i}+e y_{i}=\alpha_{i}$ for each of the four points $\left(x_{i} ; y_{i} ; 1\right) \in B^{\prime}$. 
(2) let $Y=c x+e y$, then $Y_{i}:=c x_{i}+e y_{i}$ are the roots of the polynomial $R(Y)=\left(\operatorname{Resultant}\left(Q(x,(Y-c x) / e, 1), Q_{p}^{\prime}(x, Y-c x) / e, 1\right), x\right)$. So by definition, in affine coordinates, $J=R(c x+e y)$.

4.12. Proposition. The plane quartics $E^{\prime} T_{p}\left(Q_{p}^{\prime}\right), Q_{p}^{\prime 2}, J$ lie on the same pencil.

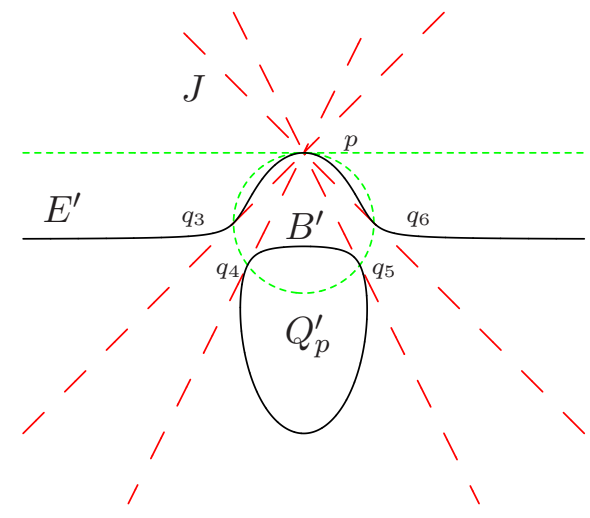

Proof. By the definition of $J, Q_{p}^{\prime}$ we have:

$$
Q_{p}^{\prime 2} \cdot J=2\left(B^{\prime}+4 p\right), \quad Q_{p}^{\prime 2} \cdot\left(E^{\prime}+T_{p}\left(Q_{p}^{\prime}\right)\right)=2\left(\left(B^{\prime}+2 p\right)+2 p\right) .
$$

So the three quartics belongs to the same pencil.

4.13. The curve $Q^{\prime}$. We compute $Q^{\prime}$ using Proposition 4.10 and symmetry. To compute the conic $Q_{p}$, one notes that $Q_{p} \cdot E=2 p+B$. By definition of $B$ in 2.16, $b \in B$ if and only if $T_{b}(E) \cdot E=2 b+p$. It is then classical (see for instance Salmon Sal]) that $Q_{p}$ is the polar conic of $p$ with respect to $E$ - recall that if $p=\left(x_{0} ; y_{0} ; z_{0}\right) \in E$ then the polar conic of $p$ with respect to $E$ is given by the equation $x_{0} E_{x}+y_{0} E_{y}+z_{0} E_{z}=0$.

4.14. To complete the calculation of $\left(C^{\prime}, \mathcal{L}^{\prime}\right)$ from $(C, \mathcal{L})$ we still have to make a final choice - the partition of the set $B^{\prime}$ to the two pairs $\left\{\left\{q_{3}, q_{4}\right\},\left\{q_{5}, q_{6}\right\}\right\}$. Geometrically, this is the choice of the singular conic $\overline{q_{3} q_{4}} \cup \overline{q_{5} q_{6}}$ among the three singular conics in the pencil spanned by the conics $Q, Q_{p}^{\prime}$. We start with a lemma on the symmetric situation:

4.15. Lemma. The following equality holds:

$$
\overline{q_{3}^{\prime} q_{4}^{\prime}} \cap \overline{q_{5}^{\prime} q_{6}^{\prime}}=p+\alpha_{E}=o,
$$

where the addition is in $\operatorname{Pic}(E)$.

Proof. To see the first equality, note that $2 q_{i}^{\prime}+p$ is a line section of $E \subset\left|K_{C}\right|^{*}$ for all $i=3, \ldots, 6$. By the proof of Theorem 2.17 and the symmetry on the construction, we also have $q_{3}^{\prime}-q_{4}^{\prime}=q_{5}^{\prime}-q_{6}^{\prime}=\alpha_{E}$. Setting $\tilde{o}:=p+\alpha_{E}$ we see that $q_{3}+q_{4}+\tilde{o}=2 q_{3}+p$ and $q_{5}+q_{6}+\tilde{o}=2 q_{5}+p$ are both line sections. To see the second equality, note that $T_{p} E \cap T_{o} E$ lies on $E$, which means that $\gamma:=p-o$ is in $\operatorname{Pic}(E)[2]$. However, by a monodromy argument on maximal isototropic flags on $\operatorname{Jac}\left(C^{\prime}\right)[2]$ containing $L_{2}^{\prime}$, we have $\gamma=\alpha_{E}$. 
4.16. In order to find an equation for $\left(C^{\prime}, \alpha^{\prime}\right)$ in the form $A_{3}^{\prime 2}=A_{1}^{\prime} A_{2}^{\prime}$, we apply projective transformation to mimic the form of the couple $(E, Q)$. Denote by $T$ a projective transformation of $\left|K_{C}\right|^{*}$ that sends $q_{1}^{\prime}, q_{2}^{\prime}$ and $\overline{q_{3} q_{4}} \cap \overline{q_{5} q_{6}}$ to the points $(1 ; 0 ; 0),(0 ; 1 ; 0)$ and $(0 ; 0 ; 1)$ respectively. Denote $T\left(E^{\prime}\right)=\left(e_{1}, \ldots, e_{10}\right)$ and $T\left(Q^{\prime}\right)=\left(d_{1}, \ldots, d_{6}\right)$. Finally define $T_{2}$ to be the transformation which operates by a multiplication of the $x$-axis by $\sqrt{e_{2} / e_{9}}$, and a multiplication of the $y$-axis by $\sqrt{e_{4} / e_{6}}$.

4.17. Theorem. The coordinates of the quadrics forms $T\left(A_{1}^{\prime}\right), T\left(A_{2}^{\prime}\right)$ are given by

$$
\left(0,0,0, e_{4} / e_{6}, 0,-1\right) \quad\left(e_{2} / e_{9}, 0,0,0,0,-1\right),
$$

while the coordinates of the quadric form $T\left(A_{3}^{\prime}\right)$ are given by

$$
\frac{\left(e_{2} e_{6}\left(e_{2} d_{3}-e_{3} d_{2}\right), 2 e_{2} e_{3} e_{4} d_{6}, 2 e_{2}^{2} e_{6} d_{6}, e_{4}\left(e_{2} e_{6} d_{5}-e_{3} e_{9} d_{2}\right)\right.}{, 2 e_{2} e_{4} e_{6} d_{6},-e_{6}\left(-e_{2} e_{5} d_{6}+e_{2} e_{6} d_{5}+e_{2} e_{9} d_{3}-2 e_{3} e_{9} d_{2}\right)}
$$

Proof. Let us assume that we have taken $A_{1}^{\prime}$ (resp. $\left.A_{2}^{\prime}\right)$ such that $T_{2} \circ T\left(A_{1}^{\prime}\right)$ (resp. $\left.T_{2} \circ T\left(A_{1}^{\prime}\right)\right)$ is the conic $y^{2}-z^{2}$ (resp. $\left.x^{2}-z^{2}\right)$. Let $T_{2} \circ T\left(A_{3}^{\prime}\right)=$ $\left(a^{\prime}, b^{\prime}, c^{\prime}, d^{\prime}, e^{\prime}, f^{\prime}\right)$. By Theorem 4.5] if we call $\left(E_{2}, Q_{2}\right)$ the data $(E, Q)$ associated to these transformations of $A_{1}^{\prime}, A_{2}^{\prime}, A_{3}^{\prime}$, we have

$$
\begin{aligned}
& E_{2}=\left(0 ; c^{\prime} ; b^{\prime} ; 0 ; e^{\prime} ; 2\left(a^{\prime}+d^{\prime}+f^{\prime}\right) ; e^{\prime} ; 0 ; b^{\prime} ; c^{\prime} ; 0\right), \\
& Q_{2}=\left(0 ; c^{\prime} e^{\prime}\left(b^{\prime 2}-1+4 a^{\prime} d^{\prime}\right)-2 b^{\prime}\left(c^{\prime 2} d^{\prime}+e^{\prime 2} a^{\prime}\right) ;\right. \\
& \quad b^{\prime}\left(-2 b^{\prime} c^{\prime} d^{\prime}-e^{\prime}+b^{\prime 2} e^{\prime}\right)+2 a^{\prime}\left(b^{\prime 2} c^{\prime}+c^{\prime} e^{2}-2 b^{\prime} e^{\prime} f^{\prime}\right)-4 a^{2} b^{\prime} e^{\prime} ; 0 ; \\
& b^{\prime 3} c^{\prime}+2 c^{\prime 2} d^{\prime} e^{\prime}+2 b^{\prime 2}\left(d^{\prime}-a^{\prime}\right) e^{\prime}-b^{\prime} c^{\prime}\left(1+4 d^{\prime 2}+4 d^{\prime} f^{\prime}\right) ; \\
& \left.c^{\prime 2} e^{\prime 2}+b^{\prime 2}\left(c^{\prime 2}+e^{\prime 2}\right)-2 b^{\prime} c^{\prime} e^{\prime}\left(a^{\prime}+d^{\prime}+f^{\prime}\right)\right) .
\end{aligned}
$$

If we let $T_{2} \circ T\left(E^{\prime}\right)=(0, \hat{c}, \hat{b}, \hat{e}, \hat{g}, \hat{e}, 0, \hat{b}, \hat{c}, 0)$ and $T_{2} \circ T\left(Q^{\prime}\right)=\left(0, \delta_{1}, \delta_{2}, 0, \delta_{3}, \delta_{4}\right)$, there exists a constant $\xi$ such that

$$
b^{\prime}=\hat{b} / \xi, \quad c^{\prime}=\hat{c} / \xi, \quad e^{\prime}=\hat{e} / \xi, \quad a^{\prime}+d^{\prime}+f^{\prime}=\hat{g} /(2 \xi)
$$

and

$$
\begin{gathered}
\left(\begin{array}{ccc}
-2 \hat{b} \hat{c}^{2} & -2 \hat{b} \hat{e}^{2} & \hat{c} \hat{e} \\
-2 \hat{b}^{2} \hat{c} & 2 \hat{b}^{2} \hat{c}+2 c \hat{e}^{2}-2 \hat{b} \hat{e} \hat{g} & \hat{b} \hat{e} \\
2 \hat{b}^{2} \hat{e}+2 \hat{c}^{2} \hat{e}-2 \hat{b} \hat{c} \hat{g} & -2 \hat{b}^{2} \hat{e} & \hat{b} \hat{c}
\end{array}\right)\left(\begin{array}{c}
d^{\prime} \xi \\
a^{\prime} \xi \\
4 a^{\prime} d^{\prime} \xi^{2}-\xi^{2}
\end{array}\right)+ \\
\hat{b}^{2}\left(\begin{array}{c}
\hat{c} \hat{e} \\
\hat{b} \hat{e} \\
\hat{b} \hat{c}
\end{array}\right)=\frac{\hat{c}^{2} \hat{e}^{2}+\hat{b}^{2}\left(\hat{c}^{2}+\hat{e}^{2}\right)-\hat{b} \hat{c} \hat{e} \hat{g}}{\delta_{4}}\left(\begin{array}{c}
\delta_{1} \\
\delta_{2} \\
\delta_{3}
\end{array}\right) .
\end{gathered}
$$

By the geometry of the configuration and the coordinates we chose, the only solutions $a^{\prime}, d^{\prime}, \xi$ to the system above arise from solutions of the quadric $T_{2} \circ T\left(A_{3}^{\prime}\right)$. Since the quadric $A_{3}^{\prime}$ is determined up to a sign, the matrix 
equation above has only one solution, and this solution determines $a^{\prime}, d^{\prime}, \xi$ up to a choice of a sign. We apply then the transformation $T_{2}^{-1}$ to find the expression of $T\left(A_{3}\right)$ in terms of $e_{i}, d_{i}$.

Remark. Note that the transformation $T_{2}$, and the square roots $\sqrt{e_{2} / e_{9}}$, $\sqrt{e_{4} / e_{6}}$ served merely as technical aids in the proof above, and indeed vanished in the final result. The situation is different with the root we take to distinguish between $q_{1}^{\prime}, q_{2}^{\prime}$. Recall that when performing the trigonal construction, one has to take a degree 2 field extension in order to construct $W^{\prime}$ from the tower $Z^{\prime} / X^{\prime} / \mathbb{P}^{1}$, and one has to construct $W^{\prime}$ in order to construct $C^{\prime}$. Since after distinguishing between $q_{1}^{\prime}, q_{2}^{\prime}$ we can construct $C^{\prime}$, the root we take when we distinguish between these points generate the field extension of the function field of $W^{\prime}$ over the function field of $Z^{\prime}$. This carries little significance when working over an algebraically closed field, but when working over a non-algebraically closed field, it reflects the fact that in order to find the isogeny $\operatorname{Prym}(Z / X) \cong \operatorname{Jac}(C)$ we may have to make a degree 2 field extension of the base field.

4.18. Corollary. Let $\mathcal{M}$ be the moduli of $a, b, c, d, e, f$ and a root of the cubic form (in $t$ ) Hessian $\left(t Q_{p}^{\prime}+Q\right)$, then:

- The space $\mathcal{M}$ is birational to a finite cover of the moduli of $C, \mathcal{L}$ with monodromy group naturally isomorphic to $D_{4}$.

- The map $T_{2} \circ T$ is defined globally over $\mathcal{M}$. Moreover as a map on quadrics in $x, y, z$ with parameters in $\mathcal{M}$, the map $T_{2} \circ T$ is an involution that lifts the involution $(C, \mathcal{L}) \longrightarrow\left(C^{\prime}, \mathcal{L}^{\prime}\right)$.

- Using affine coordinates on $\left|K_{C}\right|^{*}$ (by setting $z=1$ ) the map $\bar{k}$ is given by the formula

$$
T_{2} \circ T\left(\frac{l d x}{\partial\left(A_{1}^{\prime} A_{2}^{\prime}-A_{3}^{\prime 2}\right) / \partial y}\right)= \pm \bar{k}^{-1}\left(\frac{l d x}{\partial\left(A_{1} A_{2}-A_{3}^{2}\right) / \partial y}\right),
$$

where $l$ is any linear form.

Proof. The first assertion follows from the choice of coordinates we use (see Theorem 4.5), and the fact that the singular conics in the pencil of conics spanned by $Q_{p}^{\prime}, Q$ are in 1-1 correspondence with the roots of the cubic $\operatorname{Hessian}\left(t Q_{p}^{\prime}+Q\right)$. The dihedral group is the symmetry group acting on the nested partition of linear forms $\{\{x-z, x+z\},\{y-z, y+z\}\}$.

The second assertion follows from the definition of $T$ and $T_{2}$, and from Theorem 4.17

It is well known that a basis of regular differentials on a genus 3 non hyperelliptic curve $C$ can be given by $\left(\frac{l d x}{\partial(C) / \partial y}\right)$. With the identifications we have made during the construction on the coordinates $(x ; y ; z),\left(x^{\prime} ; y^{\prime} ; z^{\prime}\right)$ (see 4.1), the map $\bar{k}$ with this choice of bases is given by the transformation $\left(T_{2} \circ T\right)^{-1}$ up to a constant. However, since $T_{2} \circ T$ is an involution on $\mathcal{M}$, the square of this constant is 1 . 
4.19. Our final objective in this section is to show how one iterates the construction. Following 2.5. we denote with ${ }^{\sim}$ the objects related to $\left(C^{\prime}, \tilde{\mathcal{L}}\right)$. Our first task is to find an $\tilde{\alpha}$. By our analysis of the symplectic pairings in Proposition 2.4 and in 2.16 we have

$$
\begin{array}{llr}
\# D_{\alpha^{\prime}}^{-1}(\tilde{\alpha}) \cap\left\{q_{1}^{\prime}, q_{2}^{\prime}\right\}=0 & \Longleftrightarrow & \tilde{\alpha} \in L_{2}^{\prime \perp} \backslash L_{2}^{\prime}, \\
\# D_{\alpha^{\prime}}^{-1}(\tilde{\alpha}) \cap\left\{q_{5}^{\prime}, q_{6}^{\prime}\right\}=1 & \Longleftrightarrow & \tilde{\alpha} \notin L_{3}^{\prime} .
\end{array}
$$

So we can assume that $D_{\alpha^{\prime}}^{-1}(\tilde{\alpha})=\left\{q_{3}^{\prime}, q_{5}^{\prime}\right\}$. The situation can be represented as follows :

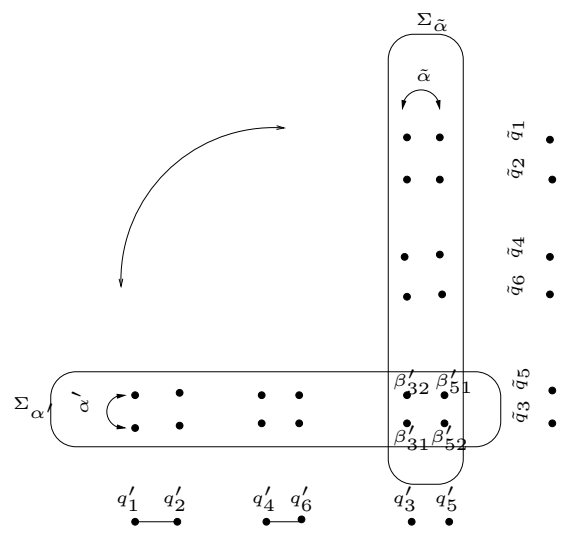

Thus, the four bitangents $\beta_{31}^{\prime}, \beta_{32}^{\prime}$ (lying over $\left.q_{3}^{\prime}\right) \beta_{51}^{\prime}, \beta_{52}^{\prime}$ (lying over $q_{5}^{\prime}$ ) in $\Gamma_{\alpha^{\prime} \oplus \alpha}=\Sigma_{\alpha^{\prime}} \cap \Sigma_{\tilde{\sigma}}$ (Proposition [2.3) can be grouped as $\left(\beta_{31}^{\prime}, \beta_{52}^{\prime}\right.$ ) and $\left(\beta_{51}^{\prime}, \beta_{32}^{\prime}\right)$ to give a possible $\tilde{\alpha}$ (the other grouping correspond to an $\left.\tilde{\alpha}+\alpha^{\prime}\right)$. Let us denote $\tilde{q}_{3}=\beta_{31}^{\prime} \cap \beta_{52}^{\prime}$ and $\tilde{q}_{5}=\beta_{51}^{\prime} \cap \beta_{32}^{\prime}$. Let $\tilde{E}, \tilde{Q}$ be the cubic and conic associated to $\left(C^{\prime}, \tilde{\alpha}\right)$. We denote $\left\{\tilde{q}_{i}\right\}_{i=1}^{6}=\tilde{Q} \cap \tilde{E}$.

The second step is to find $\tilde{L}_{2}$. By Proposition 2.4. a maximal isotropic group which contains $\alpha^{\prime}$ (resp. $\tilde{\alpha}$ ) is equivalent to the partition of $\Sigma_{\alpha^{\prime}} / \alpha^{\prime}$ (resp. $\left.\Sigma_{\tilde{\alpha}} / \tilde{\alpha}\right)$ in three pairs. There are three different maximal isotropic spaces containing $\alpha^{\prime} \oplus \tilde{\alpha}$, given by the non-zero points in $\left(\alpha^{\prime} \oplus \tilde{\alpha}\right)^{\perp} /\left(\alpha^{\prime} \oplus \tilde{\alpha}\right)$. These maximal flags are in bijection with partitions two pairs of the points $\left\{q_{1}^{\prime}, q_{2}^{\prime}, q_{4}^{\prime}, q_{6}^{\prime}\right\}$, and also with partition to pairs of the points $\left\{\tilde{q}_{1}, \tilde{q}_{2}, \tilde{q}_{4}, \tilde{q}_{6}\right\}$. One of the three maximal isotropic groups containing $\tilde{\alpha} \oplus \alpha^{\prime}$ is $\tilde{\alpha} \oplus L_{2}^{\prime}$, the two others corresponds to $\alpha^{\prime} \oplus \tilde{L}_{2}$ for the two different choices of $\tilde{L}_{2}$. Thus, in order to choose $\tilde{L}_{2}$ we first choose the maximal isotropic group $\alpha^{\prime} \oplus \tilde{L}_{2}$, and in making this choice, we exclude the partition corresponding to the group $\tilde{\alpha} \oplus L_{2}^{\prime}$.

The partition of $\left\{q_{1}^{\prime}, q_{2}^{\prime}, q_{4}^{\prime}, q_{6}^{\prime}\right\}$ which correspond to $\alpha^{\prime} \oplus \tilde{L}_{2}$ is simply the partition $\left\{q_{1}^{\prime}, q_{2}^{\prime}\right\},\left\{q_{4}^{\prime}, q_{6}^{\prime}\right\}$, but in order to proceed we will have to find the corresponding partition of $\left\{\tilde{q}_{1}, \tilde{q}_{2}, \tilde{q}_{4}, \tilde{q}_{6}\right\}$. To do this we will describe explicitly the natural isomorphism between the three partitions to two pairs of these 4-tuples. This isomorphism is geometric in nature, and to describe it we will interpret these partitions as singular conics defined by the partitions 
To describe the isomorphism we need some more notations : if $\alpha_{1}, \alpha_{2} \in$ $\operatorname{Jac}\left(C^{\prime}\right)[2]$ such that $\left\langle\alpha_{1}, \alpha_{2}\right\rangle=0$, one denote by $A_{3, \alpha_{i}}, E_{\alpha_{i}}, Q_{\alpha_{i}}, p\left(\alpha_{i}\right)$ the elements $A_{3}, E, Q, p$ relative to the construction starting from $\left(C^{\prime}, \alpha_{i}\right)$. Also we denote by $E_{\alpha_{i} \oplus \alpha_{j}}$ and $Q_{p\left(\alpha_{i}\right), \alpha_{j}}$ the curve $E^{\prime}$ and the conic $Q_{p}^{\prime}$ constructed from the data $\left(C^{\prime}, \alpha_{i} \subset \alpha_{i} \oplus \alpha_{j}\right)$ and by ' the symmetric constructions. Note that

$$
E_{\alpha_{i} \oplus \alpha_{j}}=E_{\left(\alpha_{i} \oplus \alpha_{j}\right)^{\perp} / L}^{\prime}=E_{\alpha_{j} \oplus \alpha_{i}}
$$

for any maximal isotropic group $L$ containing $\alpha_{i} \oplus \alpha_{j}$. However, $Q_{p\left(\alpha_{i}\right), \alpha_{j}} \neq$ $Q_{p\left(\alpha_{j}\right), \alpha_{i}}$.

4.20. Lemma. The identification of a singular conic through $\left\{q_{1}^{\prime}, q_{2}^{\prime}, q_{4}^{\prime}, q_{6}^{\prime}\right\}$ (resp. $\left\{\tilde{q}_{1}, \tilde{q}_{2}, \tilde{q}_{4}, \tilde{q}_{6}\right\}$ ) with a root of

$$
\operatorname{Hessian}\left(Q_{\alpha^{\prime}}+u Q_{p\left(\alpha^{\prime}\right), \tilde{\alpha}}\right)\left(\text { resp. Hessian }\left(Q_{\tilde{\alpha}}+u Q_{p(\tilde{\alpha}), \alpha^{\prime}}\right)\right)
$$

defines a natural transformation $\mu: \mathbb{P}^{1} \rightarrow \mathbb{P}^{1}$ which fixes $\infty$ and maps the two triple of roots bijectively.

Proof. We have $E_{\alpha^{\prime} \oplus \tilde{\alpha}}=E_{\tilde{\alpha} \oplus \alpha^{\prime}}$. Let $t$ be the translation on $E_{\tilde{\alpha} \oplus \alpha^{\prime}}$ by $p(\tilde{\alpha})-p\left(\alpha^{\prime}\right)$. Let $s_{Q_{p\left(\alpha^{\prime}\right), \tilde{\alpha}}}, s_{Q_{\alpha^{\prime}}}$ (resp. $\left.s_{Q_{p(\tilde{\alpha}), \alpha^{\prime}}}, s_{Q_{\tilde{\alpha}}}\right)$ denote the sections of the bundle of $E_{\alpha^{\prime} \oplus \tilde{\alpha}}$ defined by the divisor $q_{1}^{\prime}+q_{2}^{\prime}+q_{4}^{\prime}+q_{6}^{\prime}$ (resp. $\left.\tilde{q}_{1}, \tilde{q}_{2}, \tilde{q}_{4}, \tilde{q}_{6}\right)$ and corresponding to the subscript objects. Since $Q_{p\left(\alpha^{\prime}\right), \tilde{\alpha}}\left(\operatorname{resp} . Q_{p(\tilde{\alpha}), \alpha^{\prime}}\right)$ is the polar conic of $p\left(\alpha^{\prime}\right)$ (resp. $p(\tilde{\alpha})$ ), there exists $a \in K$ such that

$$
t^{*}\left(s_{Q_{p\left(\alpha^{\prime}\right), \tilde{\alpha}}}\right)=a s_{Q_{p(\tilde{\alpha}), \alpha^{\prime}}} .
$$

Thus $t$ maps the points $\left\{q_{1}^{\prime}, q_{2}^{\prime}, q_{4}^{\prime}, q_{6}^{\prime}\right\}$ onto $\left\{\tilde{q}_{1}, \tilde{q}_{2}, \tilde{q}_{4}, \tilde{q}_{6}\right\}$. Since $t^{*}\left(s_{Q_{\alpha^{\prime}}}\right)$ contains the points $\left\{\tilde{q}_{1}, \tilde{q}_{2}, \tilde{q}_{4}, \tilde{q}_{6}\right\}$, there are also two constants $b, c$ such that $t^{*}\left(s_{Q_{\alpha^{\prime}}}\right)=b s_{Q_{\tilde{\alpha}}}+c s_{Q_{p(\tilde{\alpha}), \alpha^{\prime}}}$. Thus any section in the pencil $s_{Q_{\alpha^{\prime}}}+$ $u s_{Q_{p\left(\alpha^{\prime}\right), \tilde{\alpha}}}$ is map through $t$ on $b s_{Q_{\tilde{\alpha}}}+(a u+c) s_{Q_{p(\tilde{\alpha}), \alpha^{\prime}}}$. Hence there is an affine transformation $\mu$ which maps a conic $Q_{\alpha^{\prime}}+u Q_{p\left(\alpha^{\prime}\right), \tilde{\alpha}}$ to $Q_{\tilde{\alpha}^{\prime}}+\mu(u) Q_{p(\tilde{\alpha}), \alpha^{\prime}}$. In particular, a singular conic is mapped to a singular conic which means that $\mu$ maps the three roots of $\operatorname{Hessian}\left(Q_{\alpha^{\prime}}+u Q_{p\left(\alpha^{\prime}\right), \tilde{\alpha}}\right)$ to the three roots of $\operatorname{Hessian}\left(Q_{\tilde{\alpha}}+u Q_{p(\tilde{\alpha}), \alpha^{\prime}}\right)$.

To identify the transformation $\mu$, we work on the generic case $C^{\prime}$ given by $\left(a^{\prime}, b^{\prime}, c^{\prime}, d^{\prime}, e^{\prime}, f^{\prime}\right)$ and we are looking for a continuous affine transformation. We assume that the bitangents $\beta_{31}^{\prime}, \beta_{32}^{\prime}, \beta_{51}^{\prime}, \beta_{52}^{\prime}$ are $y-z, y+z, x-z, x+z$ respectively. Denote by

$$
T:=\frac{1}{2}\left(\begin{array}{rrr}
-1 & 1 & 2 \\
1 & -1 & 2 \\
1 & 1 & 0
\end{array}\right)
$$

the transformation which sends $y-z, y+z, x-z, x+z$ to $y-z, x+z, x-z, y+z$ respectively. This projective transformation defines a linear transformation 
$T_{\tilde{\alpha}}$ on the coefficients of $A_{3, \alpha^{\prime}}$ given by $\left(a^{\prime}, b^{\prime}, c^{\prime}, d^{\prime}, e^{\prime}, f^{\prime}\right)$ maps to the coefficients of $A_{3, \tilde{\alpha}}$

$$
\begin{aligned}
& \left(\frac{1}{4}\left(a^{\prime}-b^{\prime}-c^{\prime}+d^{\prime}+e^{\prime}+f^{\prime}\right), \frac{1}{2}\left(-a^{\prime}+b^{\prime}-d^{\prime}+f^{\prime}\right), \frac{1}{2}\left(-2 a^{\prime}+c^{\prime}+2 d^{\prime}+e^{\prime}\right),\right. \\
& \left.\frac{1}{4}\left(a^{\prime}-b^{\prime}+c^{\prime}+d^{\prime}-e^{\prime}-f^{\prime}\right), \frac{1}{2}\left(2 a^{\prime}+c^{\prime}-2 d^{\prime}+e^{\prime}\right), a^{\prime}+b^{\prime}+d^{\prime}\right)
\end{aligned}
$$

Using Theorem [4.5] we can compute the different objects involved and we find

$$
\begin{aligned}
\mu(u)= & \left(2 a^{\prime}-c^{\prime}-2 d^{\prime}-e^{\prime}\right) \cdot\left(2 a^{\prime}+c^{\prime}-2 d^{\prime}+e^{\prime}\right) \\
& \cdot\left(2 a^{\prime} b^{\prime} e^{2}-4 a^{\prime} c^{\prime} d^{\prime} e^{\prime}-b^{\prime 2} c^{\prime} e^{\prime}+2 b^{\prime} c^{\prime 2} d^{\prime}+c^{\prime} e^{\prime}\right) u \\
& +2 e^{\prime} \cdot c^{\prime} \cdot a^{\prime} . \\
& \cdot\left(4 a^{\prime 2} b^{\prime}-8 a^{\prime} b^{\prime} d^{\prime}-2 a^{\prime} c^{\prime} e^{\prime}-b^{\prime} c^{\prime 2}+4 b^{\prime} d^{\prime 2}-b e^{\prime 2}-2 c^{\prime} d^{\prime} e^{\prime}+2 c^{\prime} e^{\prime} f^{\prime}\right) .
\end{aligned}
$$

Let us say a few words about the computation: we are comparing the coefficients of two monic cubic forms in $u$, under the transformation $u \mapsto$ $\left(\mu_{0} u+\mu_{1}\right)$. Thus we get the equation:

$\epsilon\left(u^{3}+a_{2} u^{2}+a_{1} u+a_{0}\right)=\left(\mu_{0} u+\mu_{1}\right)^{3}+b_{2}\left(\mu_{0} u+\mu_{1}\right)^{2}+b_{1}\left(\mu_{0} u+\mu_{1}\right)+b_{0}$.

Comparing the $u$ coefficients we get a system of equations in $\mu_{0}, \mu_{1}$ :

$$
\begin{cases}3 \mu_{1}+b_{2}-\mu_{0} a_{2} & =0, \\ 3 \mu_{1}^{2}+2 b_{2} \mu_{1}+b_{1}-\mu_{0}^{2} a_{1} & =0, \\ \mu_{1}^{3}+b_{2} \mu_{1}^{2}+b_{1} \mu+b_{0}-\mu_{0}^{3} a_{0} & =0 .\end{cases}
$$

We solve the system by finding the two solutions of the first two equations, and checking which of the two solutions solves the third equation.

4.21. By a projective transformation one can send the bitangents $\beta_{31}^{\prime}, \beta_{32}^{\prime}$, $\beta_{51}^{\prime}, \beta_{52}^{\prime}$ to $y-z, y+z, x-z, x+z$ respectively. Using the previous lemma, one can then identify the value $u_{0}$ of $u$ corresponding to $\tilde{\alpha}+L_{2}^{\prime}$ (i.e to the singular conic whose one component is $\overline{q_{1}^{\prime} q_{2}^{\prime}}$ ) and then exclude the singular conic $Q_{\tilde{\alpha}}+\mu\left(u_{0}\right) Q_{p(\tilde{\alpha}), \alpha^{\prime}}$. Let us denote this one $\overline{\tilde{q}_{1} \tilde{q}_{2}} \cup \overline{\tilde{q}_{4} \tilde{q}_{6}}$ and then $\tilde{L}_{2}$ is represented for instance by $\overline{\tilde{q}}_{4} \tilde{q}_{6}$.

The last task is to identify $\tilde{L}_{3}$ : by Lemma 2.6] it has to contain one of the points $\tilde{q}_{3}, \tilde{q}_{5}$ so it is given by any of the choice of a pair $\left\{\tilde{q}_{2}, \tilde{q}_{5}\right\}$ or $\left\{\tilde{q}_{2}, \tilde{q}_{3}\right\}$.

\section{REAl CURVES}

5.1. In this section we show that if $C$ is a real $M$ curve of genus 3 (i.e. a curve with 4 components), then the topology of the real structure induces a distinguished isotropic flag $L_{1} \subset L_{3}$ in $\operatorname{Jac}(C)[2]$ such that the curve $C^{\prime}$ is an $M$ curve. In Theorem 5.3 we establish a bijection between partitions of the four components of the curve $C$ to two pairs, and the set of full flags $\mathcal{L}$ containing the flag $L_{1} \subset L_{3}$. In [5.5] we show how to find the topologically distinguished flag $\tilde{\mathcal{L}}^{\prime}$ on the curve $C^{\prime}$ using the data $C^{\prime}, \mathcal{L}^{\prime}$ (one calculates 
$C^{\prime}, \mathcal{L}^{\prime}$ from the pair $\left.C, \mathcal{L}\right)$ by taking square roots as described in 4.19 - thus getting an iterative process - we will show that the choice of these square roots is uniquely determined by the topology. Finally, in 5.8 we describe the iterative integration algorithm.

5.2. Let $C$ be a real plane quartic with 4 components $C_{1}, C_{2}, C_{3}, C_{4}$. We denote by $\operatorname{Jac}_{\mathbb{R}}(C)$ the real part of the Jacobian of the curve $C$, and by $\mathrm{Jac}_{\mathbb{R}}(C)_{0}$ the 0 -component of $\mathrm{Jac}_{\mathbb{R}}(C)$ - see GH] p. 159. Recall that since the degree of the curve $C$ is even, each of the $C_{i}$ s is null homotopic in $\mathbb{P R}^{2}$ (see e.g. GH] ). Whence, the set $\mathbb{R P}^{2} \backslash C_{i}$ is a union of a disk and a Möbius strip. Recall also that the quotient $\operatorname{Jac}_{\mathbb{R}}(C)[2] / \operatorname{Jac}_{\mathbb{R}}(C)_{0}[2]$ is naturally isomorphic to the vector space $\mathbb{F}_{2}\left[C_{1}, C_{2}, C_{3}, C_{4}\right] / \mathbb{F}_{2}$, where $\mathbb{F}_{2}$ acts by adding $1_{\mathbb{F}_{2}}$ to all the coordinates.

Let $\left\{\left\{C_{1}, C_{3}\right\},\left\{C_{2}, C_{4}\right\}\right\}$ be a partition of the four components to two pairs. Denote by $c_{i}$ a point in the trivial component of $\mathbb{P R}^{2} \backslash C_{i}$, and choose the infinity line $l_{\infty}$ in $\mathbb{P R}^{2}$ such that the 4 points $c_{1}, c_{2}, c_{3}, c_{4}$ admit a cyclic order in $\mathbb{P R}^{2} \backslash l_{\infty}$ (formally, this means that $c_{1}, c_{2}, c_{3}, c_{4}$ sit on an ellipse in $\mathbb{P R}^{2} \backslash l_{\infty}$ in the order $\left.1,2,3,4\right)$. We assume the order induced on $c_{1}, c_{2}, c_{3}, c_{4}$ from the choice of the line $l_{\infty}$ is counter clock wise. Note that there is a natural isomorphism

$$
\mathcal{H}:=H_{1}\left(\mathbb{P R}^{2} \backslash\left\{c_{1}, c_{2}, c_{3}, c_{4}\right\}, \mathbb{F}_{2}\right) \cong \mathbb{F}_{2}\left[l_{\infty}\right] \oplus \mathbb{F}_{2}\left[C_{1}, C_{2}, C_{3}, C_{4}\right] / \mathbb{F}_{2}
$$

Since the bitangents are lines, the classes of the bitangents in $\mathcal{H}$ have non trivial $l_{\infty}$ coordinate; in fact a much stronger result holds:

5.3. Theorem. For any $i \in\{1, \ldots, 4\}$ there is a Steiner system $\Sigma_{i}$ such that the bitangents in $\Sigma_{i}$ have exactly four representatives in each of the following homology classes in $\mathcal{H}$ :

$$
l_{\infty}+C_{i}, \quad l_{\infty}+C_{i}+C_{i+1}(\bmod 4), l_{\infty}+C_{i-1}(\bmod 4)
$$

Proof. All additions of indices in the proof are modulo 4. For any $i<j$ consider a one parameter degeneration of the curve $C$ to a curve $\bar{C}_{i j}$ such that on $\bar{C}_{i j}$, the ovals $C_{i}, C_{i-1}$ are connected with a node, and the ovals $C_{j}, C_{j-1}$ are connected with a node - see the figure below. 


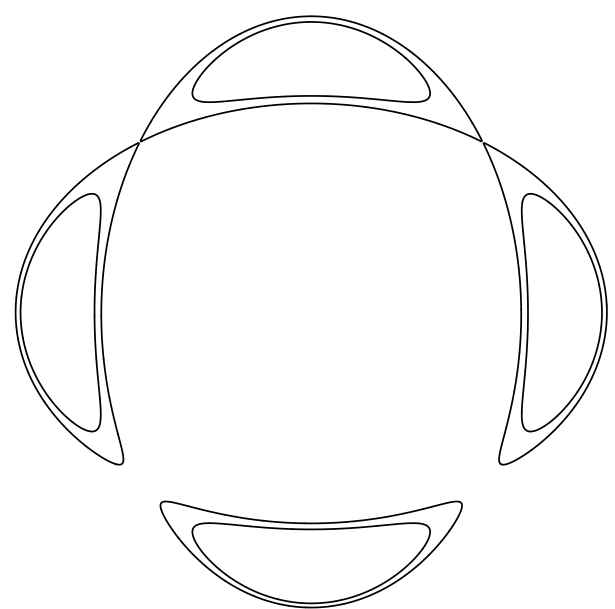

By Bea1 the degeneration of each of the two nodes degenerates a 2-torsion point in the Jacobian variety $\operatorname{Jac}(C)$; we mark these points by $\gamma_{i}, \gamma_{j}$. Under the degeneration from $C$ to $\bar{C}_{i j}$, any of the 6 pairs of bitangents in the Steiner system $\Sigma_{\gamma_{i}}$ degenerates to one double line through the node of corresponding to $\gamma_{i}$; the same property holds also for $j$. Moreover the intersection of the Steiner systems $\Sigma_{\gamma_{i}} \cap \Sigma_{\gamma_{j}}$ is then the quadrapole line through the two nodes of $\bar{C}_{i j}$. Thus by Proposition 2.4 the Weil pairing $\left\langle\gamma_{i}, \gamma_{j}\right\rangle$ is 0 , and all the bitangents in $\Gamma_{\gamma_{i} \oplus \gamma_{j}}$ have the same homology class in $\mathcal{H}$ : If $j=i+1$ this class is $l_{\infty}+C_{i}$, and if $j=i+2$ this class is $l_{\infty}+C_{i}+C_{i+1}$.

5.4. In Theorem 5.3 we identified the homology classes of 24 bitangents. Since for any $i$ there is a bitangent to $C_{i}, C_{i+1}$ in the class $l_{\infty}$, this completes the identification of the bitangents. By Proposition 2.4 we have also identified a distinguished flag given by the following partition data:

- Four bitangents in the class of $l_{\infty}$.

- Four bitangents in each of the classes $l_{\infty}, l_{\infty}+C_{1}+C_{3}, l_{\infty}+C_{2}+C_{4}$ by the combinatorial structure we described and by Proposition 2.4 this is a Steiner system.

- Four bitangents in each of the classes in $\mathcal{H}$ with non trivial line coordinate except the class $l_{\infty}+C_{1}+C_{3}$.

Finally recall (see Huil) that since $C$ is an $M$ curve, the variety $\operatorname{Jac}_{\mathbb{R}}(C)_{0}$ is naturally isomorphic to a product of any $3(=\operatorname{genus}(C))$ of the components. Since the group $\operatorname{Jac}_{\mathbb{R}}(C)_{0}[2]$ is spanned by any two subgroups of order 4 in it, and since four of the order 4 subgroups we built above sit in the product of 3 components of the curve, the maximal group in the flag we built is $\operatorname{Jac}_{\mathbb{R}}(C)_{0}[2]$. Note that the quotient $\operatorname{Jac}_{\mathbb{R}}(C) / L_{3}$ is the Jacobian of an $M$ curve if and only if this quotient has $2^{3}$ components, which means that $L_{3}=\operatorname{Jac}_{\mathbb{R}}(C)_{0}[2]$ - thus the choice of a distinguished $L_{3}$ we made above is indeed the unique choice which will enable iteration. 
5.5. To complete the description of an iterative algorithm we have to solve two problems: initiating the algorithm, and performing an iterative step. In the discussion of these problems we will apply several times the proposition below.

5.6. Proposition. The symplecto-algebraic properties of a configuration of bitangents to a real $M$-curve of genus 3 , as points on the odd part of an affine symplectic space, are determined by the homotopy classes of the bitangents in $\mathbb{P R}^{2} \backslash\left\{c_{i}\right\}_{i=2}^{4}$ and the intersection pattern of the bitangents with the components of $C$.

Proof. This follows from the following facts:

- Bitangents are continuous on families. Thus, the homology classes of bitangents in $\mathbb{R P}^{2} \backslash\left\{c_{i}\right\}_{1}^{4}$ is constant on families.

- Level structure is continuous on families.

- The moduli space of $M$-curves is irreducible.

5.7. We will apply Proposition 5.6 several times to study the configuration of bitangents arising from 4.19. We will describe real algebro-geometric data on the moduli of configurations of bitangents that defines several non-zero real algebraic functions. To show that some real configuration is associated with a distinguished flag (in the sense of Theorem [5.3) we will present one curve for which our function is positive on the distinguished configuration and negative on the others. The conceptual calculations appear below. The related numeric calculations are in $[\mathrm{LR}$.

Initiating the algorithm. To initiate the algorithm one essentially has to solve, in a Galois theory sense, the bitangents of the curve $C$. As the Galois group acting on the bitangents is generically unsolvable, this problem is generically unsolvable in radicals. However, there are still other computationally useful problems we will answer:

- Determine if on a given M-curve of genus 3 and quadrics $A_{1}, A_{2}, A_{3}$, the flag $L_{1} \subset L_{2}$ induced from $A_{1}, A_{2}, A_{3}$ is a subflag of a distinguished flag (in the sense of Theorem 5.3). To check this it suffices to verify that the curve $C$ lies in one component of $\mathbb{P R}^{2} \backslash \operatorname{Nulls}\left(A_{1} A_{2}\right)$. It suffices to check this on an infinitesimal neighborhood of the 4 bitangents determined by the conics $A_{1}, A_{2}$.

- Given an M-curve $C$ in the form $A_{1} A_{2}-A_{3}^{2}$ such that the induced flag is a subflag of a distinguished flag, mark the choice of the distinguished group $L_{3}$. Note that the quartic form $\left(x^{2}-z^{2}\right)\left(y^{2}-z^{2}\right)$ separate the real projective plane to 3 positive components and 4 negative components. Thus, if the form $A_{3}^{\prime}$, calculated as in 4.17 is purely imaginary, then the curve $C^{\prime}$ is an $\mathrm{M}$ curve, and thus the choice of $L_{3}$ is the distinguished choice. Note also that $A_{3}^{\prime}$ is purely imaginary if and only if the expression under the square root in Theorem 4.17 is negative. By Proposition [5.6] it suffice to show one example of a curve $C$ with purely imaginary $A_{3}^{\prime}$. We do this in [LR]. 
Describing the iterative step: Recall (see 4.19) that during the calculation of the iterative step in 4.19 the field extensions where geometrically described by making several times choices of the following type: given three lines $l_{1}, l_{2}, l_{3}$ and two pairs of points $p_{i 1}, p_{i 2} \in l_{i} \backslash\left(l_{i} \cap\left(l_{3-i} \cup l_{3}\right)\right)$ find a partition to two pairs of the four points $p_{11}, p_{12}, p_{21}, p_{22}$ that is not the one arising from the lines $l_{1}, l_{2}$. This choice boils down to a positivity question: we consider the pencil of conics throughput the four points $p_{11}, p_{12}, p_{21}, p_{22}$. There are three singular conics in this pencil, one of which is given by $l_{1} \cup l_{2}$. Moreover, $l_{1} \cup l_{2}$ cuts $\mathbb{P R}^{2}$ to two components, and the two nodes of the two other singular conics in the pencil, which are $\overline{p_{11} p_{21}} \cup \overline{p_{22} p_{12}}, \overline{p_{11} p_{22}} \cup \overline{p_{12} p_{21}}$ appear one in each of the two components of $\mathbb{P R}^{2} \backslash\left(l_{1} \cup l_{2}\right)$. We calculate the choices which bring us to a distinguished on $C^{\prime}$ (in the sense of Theorem 5.3 in [LR]). We plot in the figure below one step of the computation - finding the distinguished topological configuration of $\Gamma_{L_{1}^{\prime} \oplus \tilde{L}_{1}}$ (See 4.19). The set $\Gamma_{L_{2}^{\prime}}$ is plotted in dashed red lines and the set $\Gamma_{\tilde{L}_{1} \oplus L_{1}^{\prime}}$ is plotted in blue dotted lines.

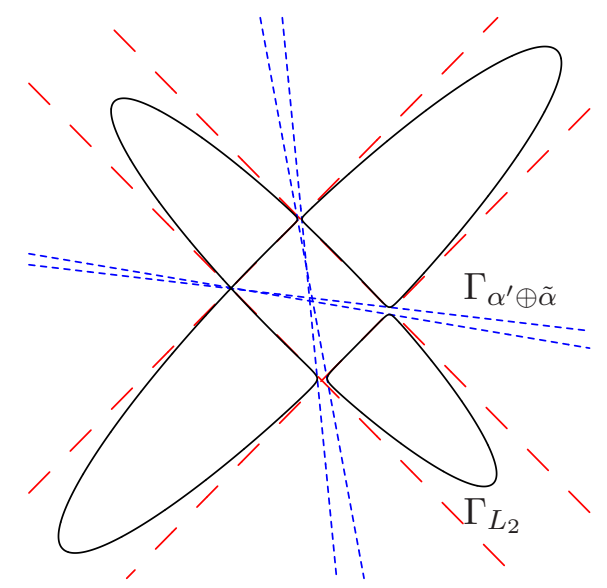

5.8. We conclude this section with a description of the integration algorithm. By Hui] the 0 component of the real Jacobian of the curve $C$ is a product of any 3 of the components of $C$. Thus, real projective cycles on the Jacobian of $C$ are homologous to sums of $C_{i}$ s. By the same argument, these sums map under our construction to sums of the components of $C^{\prime}$. Considering the theta function of $C$ we see that when the AGM algorithm is applied iteratively, the distances between the bitangents in each of the distinguished 4-tuples are decreasing exponentially. i.e., the limit curve of this process is a union of 4 lines in $\mathbb{P R}^{2}$. Using this method and Corollary 4.18 we reduced the calculation of integrals of cycles on $\operatorname{Jac}_{\mathbb{R}}(C)$ to calculation of integrals of rational functions on line segments.

In the figure below, which is computed in the final step of [LR, we plot two iterations of our algorithm, where the canonical classes of the curves $C, C^{\prime}$, and the next curve in the iterative process are identified. 


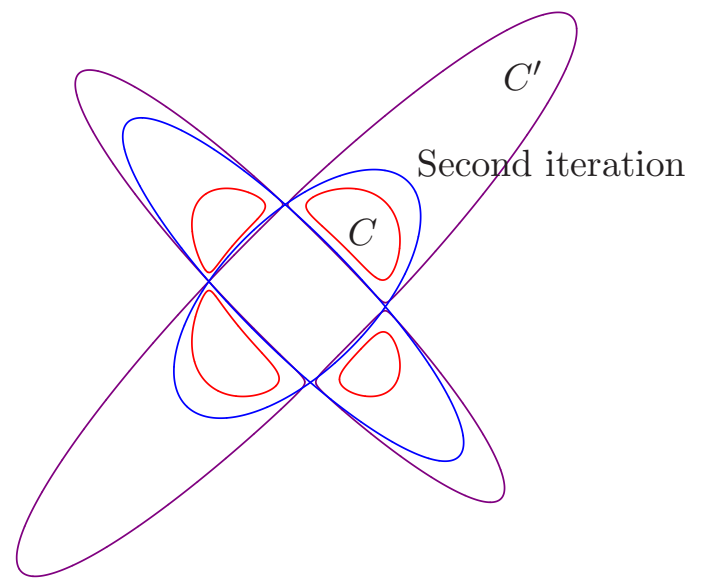

\section{REFERENCES}

[ACGH] Arbarello, E.; Cornalba, M.; Griffiths, P. A.; Harris, J. Geometry of algebraic curves. Vol. I. Grundlehren der Mathematischen Wissenschaften, 267. SpringerVerlag, 1985.

[Bea1] Beauville, A. Prym varieties and the Schottky problem. Invent. Math. 41 (1977), no. 2, 149-196.

[Bea2] Beauville, A. Sous-variétés spéciales des variétés de Prym. Compositio Math. 45 (1982), no. 3, 357-383.

[BB] Borwein, J. M.; P. B. Borwein, P. B. Pi and the AGM: A Study in Analytic Number Theory and Computational Complexity. A Wiley-Interscience Publication. John Wiley \& Sons, Inc., 1988.

[BM] Bost, J.-B.; Mestre, J.-F. Moyenne Arithmetico-geometrique et Périodes des Courbes de genere 1 et 2. Gaz. Math. No. 38 (1988), 36-64.

[Cob] Coble, A. Algebraic geometry and Theta functions. Revised printing. American Mathematical Society Colloquium Publication, vol. X American Mathematical Society, 1961.

[Cox] Cox, D. A. The arithmetic-geometric mean of Gauss. Enseign. Math. (2) 30 (1984), no. 3-4, 275-330.

[Dol1] Dolgachev, I. Rationality of $\mathcal{R}_{3}$. Unpublished notes, available online at http://www.math.lsa.umich.edu/ idolga/preprints.html

[Dol2] Dolgachev, I. Classical Algebraic geometry. A manuscript in progress. Available online at http://www.math.lsa.umich.edu/ idolga

[Don] Donagi, R. The fibers of the prym map. Curves, Jacobians, and abelian varieties (Amherst, MA, 1990), 55-125, Contemp. Math., 136, Amer. Math. Soc., 1992. Available online at alg-geom/9206008

[DL] Donagi, R.; Livné, R. The arithmetic-geometric mean and isogenies for curves of higher genus. Ann. Scuola Norm. Sup. Pisa Cl. Sci. (4) 28 (1999), no. 2, 323-339. Available online at alg-geom/9712027

[GH] Gross, B.; Harris, J. Real algebraic curves. Ann. Sci. École Norm. Sup. (4) 14 (1981), no. 2, 157-182. Available online at http://archive.numdam.org/article/ASENS_1981_4_14_2_157_0.pdf

[Har] Harris, J. Galois groups of enumerative problems. Duke Math. J., 46 (1979), no. 4, 685-724.

[Har] R. Hartshorne Algebraic Geometry Springer Verlag, GTM 52. (1977) 
[Hui] Huisman, J. A group law on smooth real quartics having at least 3 real branches. J. Thor. Nombres Bordeaux 14 (2002), 249-256. Available online at http://fraise.univ-brest.fr/ huisman/recherche/publications/jq.html

[Hum] Humbert, G. Sur la transformation ordinaire des fonctions abeliennes. J. de math. (5) 7 (1901).

[Jor] Jordan, M.C. Traité des substitutions et des équations algébriques. Gauthier-Villars, Paris, 1870 .

[Kon] Konigsberger, L. Uber die transformation der Abelschen Functionen erster ordnung. J. reine angew. Math. 64 (1865) 17-42.

[Leh] Lehavi, D. A smooth plane quartic can be reconstructed from its bitangents. Isr. J. Math. 146, 371-379 (2005). Available online at math.AG/0111017

[LL] R. Lercier \& D. Lubicz : A quasi quadratic time algorithm for hyperelliptic curve point counting. Available online at http://www.medicis.polytechnique.fr/ lercier/preprints/riemann.pdf

[LR] Lehavi, D; Ritzenthaler, C. A proof of the arithmetic geometric mean formula in genus 3 - a computer program. Availbale online at http://www.math.ohio-state.edu/ dlehavi

[Mes] Mestre, J.-F. Lettre adressée à Gaudry et Harley, Décembre 2000. Available online at http://www.math.jussieu.fr/ ${ }^{\text {mestre/ }}$

[Mum] Mumford, D. Prym varieties I. Contributions to analysis (a collection of papers dedicated to Lipman Bers), pp. 325-350. Academic Press, 1974.

[Pan] S. Pantazis Prym Varieties and the Geodesic Flow on $S O(n)$ Math. Ann. 273 p. 297-315 (1986).

[Rec] Recillas, S. Jacobians of curves with $g_{4}^{1}$ 's are the Pryms of trigonal curves. Bol. Soc. Mat. Mexicana (2) 19 (1974), no. 1, 9-13.

[Ric] Richelot, F. De transformatione integralium Abelianorum primi ordinis comentatio. J. reine angew. Math. 16 (1837) 221-341.

[Rit] Ritzenthaler, C. Problèmes arithmt́iques relatifs à certaines familles de courbes sur les corps nis. Thèse de Doctorat, Université Paris 7. Available online at http://www.math.jussieu.fr/ ritzenth/

[Sal] G. Salmon : A treatise on the higher plane curves, troisième édition, Chelsea, (1879).

Mathematics Department, Princeton University, Fine Hall, Washington Road, Princeton, NJ 08544, USA

E-mail address: dlehavi@math.princeton.edu

Institut de Mathématiques de Luminy, 163 Avenue de Luminy, Case 907, 13288 Marseille, France

E-mail address: ritzenth@math.jussieu.fr 